\title{
Haemophilia B: database of point mutations and short additions and deletions-fourth edition, 1993
}

\author{
F.Giannelli, P.M.Green, K.A.High', S.Sommer ${ }^{1}$, M.-C.Poon ${ }^{3}$, M.Ludwig ${ }^{4}$, R.Schwaab ${ }^{4}$, \\ P.H.Reitsma ${ }^{5}$, M.Goossens ${ }^{6}$, A.Yoshioka $^{7}$ and G.G.Brownlee ${ }^{8}$ \\ Paediatric Research Unit, Guy's Tower, London Bridge, London SE1 9RT, UK, 'The Children's \\ Hospital of Philadelphia, Division of Hematology, Room 5132, 34th Street \& Civic Centre Boulevard, \\ Philadelphia, PA, ${ }^{2}$ Mayo Clinic, 200 Southwest First Street, Rochester, MN 55905, USA, 3 University of \\ Calgary, Foothills Hospital, Calgary, AB T2N 2T9, Canada, ${ }^{4}$ Institute of Clinical Biochemistry, \\ Sigmund-Freud-Strasse 25, 5300 Bonn 1, and Institute of Experimental Haematology and Blood \\ Transfusion, University of Bonn, Germany, ${ }^{5}$ Hemostasis and Thrombosis Research Unit, University \\ Hospital, PO Box 9600, 2300 RC Leiden, The Netherlands, ${ }^{6}$ National Institute of Health and Medical \\ Research, 51 Ave du Maréchal de Lattre de Tassigny, 94010 Creteil, France, ${ }^{7}$ Department of \\ Pediatrics, Nara Medical College, Kashihara City, Nara, Japan and ${ }^{8}$ Sir William Dunn School of \\ Pathology, University of Oxford, South Parks Road, Oxford OX1 3RE, UK
}

The data base below lists known point mutations and short deletions and additions in the factor IX gene, causing the bleeding disorder haemophilia B or Christmas disease (for reviews, see Brownlee 1988, Giannelli 1989, Thompson 1990, Green et al 1991a) These mutations result in a defective clotting factor IX-a glycoprotein of 415 amino acid residues normally present in plasma and an essential component of the clotting cascade. The disease is an $\mathrm{X}$-linked inherited recessive disorder affecting 1 in about 30,000 males and only very rarely females.

The purpose of this database is to update last year's one (Giannelli et al, 1992) by collecting in an accessible, summary form, molecular data on the causative mutations of haemophilia $B$ patients worldwide. It is not intended to replace primary publications although it does contain a significant amount of unpublished work. We have continued our database numbering system (Giannelli et al (1991) giving all patients a unique Patient Identity Number (PIN or ID number). As in previous years, we have included repeat observations of the same mutation, as well as molecularly unique mutations, but in order to keep the main table to a manageable size we have deleted from the table all the repeats of molecular events that were reported more than 5 times. Such events are entered in the table only once but the FIX:C and FIX:Ag entries show the average of the factor IX values associated with these mutations. The comments relevant to each group of mutations are summarised and a full list of references and PIN numbers is given. The central coordinators will keep the data relative to the excluded mutations in a separate file that will be available on request.

The factor IX gene lies on the long arm of the X chromosome at Xq27 and its entire sequence of $33 \mathrm{~kb}$ is known (Yoshitake et al, 1985). It contains 8 exons $(\mathrm{a}-\mathrm{h})$ encoding 6 major domains of factor IX. These are: (1) exon a-a hydrophobic signal peptide which targets the protein for secretion from the hepatocyte into the blood stream. (2) exons b and $\mathrm{c}-\mathrm{a}$ propeptide and gla domain, - the latter containing $12 \gamma$-carboxyglutamyl residues. This post-translational modification is required for the correct folding and calcium binding of factor IX. (3) exon d-a type $B$, or first epidermal growth factor-like domain, which shows homology to epidermal growth factor (EGF) and, in addition, contains conserved carboxylate residues including a $\beta$ hydroxyaspartate at amino acid 64 . This domain binds an additional $\mathrm{Ca}^{2+}$ with high affinity (Handford et al, 1991). (4) exon $\mathrm{e}-\mathrm{a}$ type $A$, or second epidermal growth factor-like (EGF) domain which lacks the conserved carboxylate residues of the EGF type B domain. (5) exon $\mathrm{f}$-an activation domain, within which factor XIa cleaves twice, converting factor IX to IXa; (6) exons $\mathrm{g}$ and $\mathrm{h}$-the serine protease or catalytic domain, responsible for the proteolysis of factor $\mathrm{X}$ to $\mathrm{Xa}$. This region is homologous to other well studied serine proteases (e.g. chymotrypsin) and it is thought likely that his (221), asp (269) and ser (365), all participate in the classical catalytic mechanism.

Factor IX is initially synthesised in the liver as a precursor molecule, either 46,41 or 39 amino acids (it is not known which, although 39 is probable (Pang et al, 1990)) longer at its $\mathrm{N}$ terminus than the 415-long mature factor IX found in plasma. Processing steps occur in the hepatocyte prior to secretion and sequentially remove the hydrophobic signal peptide and the propeptide. In addition to the $\gamma$-carboxylation of the $12 \mathrm{~N}$-terminal glutamyl residues carried out by a vitamin $\mathrm{K}$-dependent carboxylase, and the partial $\beta$-hydroxylation of aspartate $64, \mathrm{~N}$ linked carbohydrate side chains are added at residues 157 and 167 and an O-linked carbohydrate at least at serine 53 and 61 (Hase et al, 1988; Nishimura et al, 1992).

There are 806 patient entries in this fourth edition of the database compared with 574 patients last year (Giannelli et al, 1992). Besides point mutants, it includes 72 short (defined as less than 20 nucleotides) deletions or additions or both, made up from 54 deletions, 15 additions and 3 examples involving both additions and deletions. There are also 13 double mutations, 1 triple mutation, 12 inhibitor patients and 7 female haemophiliacs. The list excludes the 29 patients with partial or complete gene deletions or more complex rearrangements (Thompson, 1990) 
3076 Nucleic Acids Research, 1993, Vol. 21, No. 13

Summary Table derived from main database

\begin{tabular}{|c|c|c|c|c|}
\hline Location 1 & Exon & $\begin{array}{l}\text { Nucleotide } \\
\text { number }^{3}\end{array}$ & $\begin{array}{l}\text { Number of } \\
\text { mutants }{ }^{2}\end{array}$ & $\begin{array}{l}\text { Unique } \\
\text { molecular events }{ }^{2}\end{array}$ \\
\hline $\begin{array}{l}\text { Signal peptide } \\
(-46 \text { to }-18)\end{array}$ & a & $30-116$ & 6 & 6 \\
\hline $\begin{array}{l}\text { Propeptide } \\
(-17 \text { to }-1)\end{array}$ & b & $6,326-6,375$ & 54 & 6 \\
\hline $\begin{array}{l}\text { Gla } \\
\text { (1 to 46) }\end{array}$ & $\begin{array}{l}\mathrm{b} \\
\mathrm{c}\end{array}$ & $\begin{array}{l}6,376-6,489 \\
6,678-6,701\end{array}$ & 66 & 43 \\
\hline $\begin{array}{l}\text { EGF (1st) } \\
\text { (47 to } 84)\end{array}$ & d & $10,392-10,505$ & 71 & 35 \\
\hline $\begin{array}{l}\text { EGF (2nd) } \\
\text { (85 to } 127)\end{array}$ & e & $17,669-17,797$ & 40 & 32 \\
\hline $\begin{array}{l}\text { activation } \\
\text { (128 to } 195)\end{array}$ & f & $20,363-20,565$ & 94 & 37 \\
\hline $\begin{array}{l}\text { catalytic } \\
\text { (196 to } 415)\end{array}$ & $\begin{array}{l}\mathrm{g} \\
\mathrm{h}\end{array}$ & $\begin{array}{l}30,039-30,153 \\
30,822-31,372\end{array}$ & $\begin{array}{r}45 \\
354\end{array}$ & $\begin{array}{r}24 \\
146\end{array}$ \\
\hline$\underline{\text { Subtotal }}$ & & & $\underline{730}$ & $\underline{329}$ \\
\hline Promoter & & & 21 & 13 \\
\hline Donor splice sites & & & 28 & 18 \\
\hline Acceptor splice sites & & & 19 & 15 \\
\hline Cryptic splice ${ }^{4}$ & & & 8 & 4 \\
\hline Poly(A) site & & & 0 & 0 \\
\hline Totals & & & 806 & 378 \\
\hline
\end{tabular}

1 Amino acid numbers used (Anson et al, 1984)

2 Excluding normal variants within double mutants

3 For numbering, see Yoshitake et al (1985)

4 These are possible new splice sites within exons

and others observed since. Of the 806 patients studied (see Summary Table), 378 are unique molecular events, the remainder being repeats. As is well known, many of these repeats occur at CG doublets and involve a CG $\rightarrow$ TG or CA change. As discussed before (Giannelli et al, 1991), such mutants are believed to be genuine 'hotspots' for mutation. However it is now becoming clear that the high number of repeat observations at some CG doublets (e.g. 41 examples at 31,008$)$ are caused, at least in part, by a founder effect. A founder effect is now well established (Thompson et al, 1990; Bottema et al, 1990b) for a mutation at a residue other than CGs (e.g. 30 examples at nucleotide 31,311 ) and there are many examples in the database of mutations repeating 2,3 or occasionally 4 times. Probably many but not all of these will have a common origin.

We have continued to include information in the comments section, on new (or de novo) mutations where known. In addition, we note whether the mutation occurred in the mother or in the maternal grandfather or grandmother. Two coordinators have also included the age of the parent of the child carrying, or affected by, the mutant gene. Because not all coordinators list new mutations, and such data is incomplete, this database cannot be usefully analysed for the frequency of such new mutations.

The distribution of mutants according to protein domains and control regions within the gene (see Summary Table) shows that mutations have been detected in all categories listed except the poly(A) site. Remarkably, there are now 13 molecularly unique mutants occurring within a short region of the promoter, and these are invaluable in studying gene regulation (Crossley et al, 1992). Missense mutations within exons give valuable information as to the importance of particular amino acid residues. The present list contains 234 different amino acid substitutions. Fifty three residues of factor IX show two or more amino acid substitutions. These include the active site serine (amino acid 365), and the proposed active site aspartate (amino acid 269), but none is yet known for the proposed active site histidine at amino acid 221. Mutations at 7 of the $12 \gamma$ carboxyglutamyl residues have now been detected, confirming their critical role for the function of factor IX. Amino acid substitutions have also been found at 21 of the 22 cysteines of circulating factor IX and therefore mutations have ben found that compromise each one of the disulphide bridges of the mature protein, thus confirming the importance of such structures. This year 153 missense mutations were added, of which 58 are new.

The third (1992) edition of this data base (Giannelli et al 1992) is now available from the EMBL file server. It can be obtained by sending the command GET HAEMB.DAT to NETSERV@EMBL-HEIDELBERG.DE (Internet address). A documentation file introducing the format used, which differs 
Nucleic Acids Research, 1993, Vol. 21, No. 133077

from this present data base because of restrictions imposed in transmitting data by electronic mail, is obtained by sending GET HAEMB.DOC to the same address. The format used in the 1991 File Server version of the data base is somewhat related to that used for the familiar EMBL nucleic acid sequence data base and should allow easy computer searching for particular features. The new entries in this 1993 edition of the data base will be reformatted and transferred to the EMBL file server during 1993.

The data base was compiled by the central coordinators (Giannelli and Green) from separate lists updating the previous year's list prepared by coordinators for the different countries as follows: Giannelli and Green representing the UK, Sweden and Iceland; High and Sommer representing USA; Poon representing Canada; Ludwig and Schwaab representing Germany; Reitsma representing The Netherlands; Goossens representing France; Yoshioka representing Japan; and Brownlee, the rest of the world. New data or notification of errors or omissions should be sent to the individual country coordinators. This database, including all repeated mutations, is available from individual country coordinators on floppy discs written in Wordperfect 5.1 on an IBM PS2 computer.

\section{REFERENCES}

1. Alkan M., Rodriguez Ponte M., Malik N.J. et al (1991) Nucl.Acids Res. 19: 409.

2. Anson D.S., Choo K.H., Rees D.J.G. et al (1984) EMBO J. 3: 1054-1064.

3. Attree O., Vidaud D., Vidaud M., Amselem S., Lavergne J.M. and Goossens M. (1989) Genomics 4: 266-272.

4. Bajaj S.P., Spitzer S.G., Welsh W.J., Warn-Cramer B.J., Kasper C.K., and Birktoft J.J. (1990) J.Biol.Chem. 265: 2956-61. .

5. Bentley A.K., Rees D.J.G., Rizza C. and Brownlee G.G. (1986) Cell 45: 343-348.

6. Bertina R.M., Van der Linden I.K., Mannucci P.M. Reinalda-Poot H.H., Cupers R., Poort S.R. and Reitsma P.H. (1990) J.Biol.Chem. 265: $10876-10883$

7. Bottema C.D.K., Koeberl D.D. and Sommer S.S. (1989a) Lancet (ii), $526-529$.

8. Bottema C.D.K., Ketterling R.P., Cho H.I. and Sommer S.S. (1989b) Nucl. Acids Res. 17: 10139.

9. Bottema C.D.K., Ketterling R.P., Koeberl D.D. et al (1990a) Nucl. Acids Res. 18: 1924

10. Bottema C.D.K., Koeberl D.D., Ketterling R.P., Bowie E.J.W., Taylor S.A.M., Lillicrap D., Shapiro A., Gilchrist G., and Sommer S.S. (1990b) Br.J.Haematol. 75: 212-216..

11. Bottema C.D.K., Ketterling R.P., Yoon H.S., and Sommer S.S. (1990c) Am.J.Hum.Genet. 47: 835-841.

12. Bottema C.D.K., Ketterling R.P., Ii S., Yoon, H.S., Phillips J.A. and Sommer S.S. (1991a) Am. J. Hum. Genet. 49: 820-838.

13. Bottema, C.D.K., Bottema M.J., Ketterling R.P., Yoon H.S., Janco R.L., Phillips J.A. and Sommer S.S. (1991b) Am. J. Hum. Genet. 49: 839-850.

14. Brownlee G.G. (1988) in: Recent Advances in Haematology, ed. Hoffbrand A.V. (Churchill Livingstone) vol.5, pp. 251-264.

15. Caglayan S.H., Gursel T., Guler E., Soysal T., Sommer S.S.; and Gokmen Y., Altay C., and Kirdar B., Dept. Biology, Bogazici Univ., 80815 Bebek, Istanbul, Turkey, unpublished.

16. Chan V. et al, Dept of Medicine, Univ. of Hong Kong, Queen Mary Hospital, Hong Kong, unpublished.

17. Chan V., Yip B., Tong T.M.F. et al (1991) Br. J. Haematol. 79: 63-69.

18. Chen S.H., Scott C.R., Lovrien E.W. et al (1989a) Am. J. Hum. Genetics 44: $567-569$

19. Chen S.H., Thompson A.R, Zhang M., and Scott C.R. (1989b) J. Clin. Invest. 84: 113-118.

20. Chen S.H., Zhang M., Lovrien E.W., Scott C.R. and Thompson A.R. (1991a) Hum. Genet. 87: 177-182.

21. Chen S.H., Zhang M., Thompson A.R., Bray G.L., and Scott C.R. (1991b) Nucl. Acids Res. 19:1172.

22. Costes B., et al Natiional Institute of Health \& Medical Research, 51 Av. du Maréchal de Lattre de Tassigny, Creteil, France, unpublished.
23. Crossley P.M., Winship P.R., Black A., Rizza C. and Brownlee G.G. (1989) Lancet (i): 60 .

24. Crossley P.M. and Brownlee G.G. (1990) Nature 345: 444-446.

25. Crossley M., Winship P.R., Austen D.E.G., Rizza C.R. and Brownlee G.G. (1990) Nucl.Acids Res. 18: 4633

26. Crossley M., Ludwig M., Stowell K.M., De Vos P., Olek K. and Brownlee G.G. (1992) Science 257: 277-279.

27. Damas C., Solera J., Magallon M., Coloma A., Dept. Biochem., Univ. Autonoma, Madrid, 28029, Spain, unpublished.

28. David D. et al.,National Inst. of Health, Av. Padre Cruz, P-1699, Lisbon, Portugal, unpublished.

29. Davis L.M, McGraw R.A., Ware J.L. et al (1987) Blood 69: 140-143.

30. De la Salle C., INSERM U311, CRTS, 10 Rue Spielman, 67085 Strasbourg, France, unpublished.

31. Demers D.B., Odelberg S.J. and Fisher L. (1990) Nucl. Acids Res. 18: 5575.

32. Denton P.H., Fowlkes D.M., Lord S.T., and Reisner H.M. (1988) Blood 72: $1407-1411$

33. Deutz et al., Dept. of Human Genetics, Leiden, The Netherlands, unpublished.

34. Diuguid D.L., Rabiet M.J., Furie B.C. et al (1986) Proc. Natl. Acad. Sci. (USA) 83: 5803-5807.

35. Diuguid D.L., Rabiet M.J., Furie B.C., and Furie B. (1989) Blood 74: 193-200.

36. Driscoll M.C., Bouhassira E. and Aledort L.M. (1989a) Blood 74: 737-742.

37. Driscoll M.C., Aledort L.M., Hilgartner M.W. (1989b) Blood 74: 254a (abstract) and unpublished observation.

38. Figueiredo M.S. (1993) Braz. J. Med. \& Biol. Res., in press.

39. Fraser B.M., Poon M-C. and Hoar D.I. (1992) Hum. Genet. in press.

40. Freedenberg D.L. and Black B. (1991) Thromb. Haemost. 65: 964 (abstract).

41. Freedenberg D.L., Chen S.H., and Scott R. (1989) Am J. Hum. Genetics 45 (suppl): A186 (abstract)

42. Geddes V.A., LeBonniec B.F., Louie G.V., Brayer G.D., Thompson A.R., and MacGillivray R.T.A. (1991) J.Biol.Chem. 264: 4689-4697.

43. Ghanem N., Costes B., Martin J., Vidaud M., Rothschild C., FoyerGazengel C. and Goossens M. (1993) Eur. J. Hum. Genet. in press.

44. Giannelli F., (1989) in: Baillière's Clinical Haematology, ed. Tuddenham E.G.D. (Baillière \& Tindall, London) vol.2, pp.821-848.

45. Giannelli F., Green P.M., High K.A. et al (1991) Nucl.Acids Res. 19(suppl): 2193-2219.

46. Giannelli F., Green P.M., High K.A. et al (1992) Nucl.Acids Res. 20(suppl): 2027-2063.

47. Gispert S., Vidaud M., Vidaud D., Gazengel C., Boneu B. and Goossens M. (1989) Am.J.Hum.Genet. 45 Abstract 739.

48. Green P.M., Bentley D.R., Mibashan R.S., Nilsson I.M. and Giannelli F. (1989) EMBO J. 8: 1067-1072.

49. Green P.M., Montandon A.J., Bentley D.R., Ljung R., Nilsson I.M. and Giannelli F. (1990) Nucl. Acids Res. 18: 3227-3231.

50. Green P.M., Montandon A.J., Bentley D.R. \& Giannelli F. (1991a) Blood Coagulation \& Fibrinolysis 2: 539-565.

51. Green P.M., Montandon A.J., Ljung R., Bentley D.R., Kling S.R., Nilsson I.M. and Giannelli F. (1991b) Br. J. Haematol. 78: 390-397.

52. Green P.M., Montandon A.J., Ljung R., Nilsson I.M. and Giannelli F. (1992a) Thromb. Haemost. 67: 66-69.

53. Green P.M., Mitchell V.E., Goldman E. \& Giannelli F. (1992b) Br. J. Haematol. 80, suppl.1: 16.

54. Green P.M., Montandon A.J. et al, Paediatric Research Unit, Guy's Hospital Tower, London, UK, unpublished.

55. Hall A., Chuansumrit A., Peake I.R. and Winship P.R. (1992) Br. J. Haematol. 80, suppl.1:16.

56. Hamaguchi M., Matsushita T., Tanimoto M. et al (1991) Thromb. Haemost. 65: $514-520$

57. Handford P.A., Mayhew, M., Baron, M., Winship, P.R., Campbell, I.D. \& Brownlee, G.G. (1991) Nature 351: 164-167.

58. Hase, S., Kawabata, S., Nishimura, H., Takeya, H., Sueyoshi, T., Miyata, T., Iwanaga, S., Takao, T., Shimonishi, Y. \& Ikenaka, T. (1988) J. Biochem. 104: 867-868.

59. High K.A., Stanfield-Oakley S. \& Kaspar C. (1993) Thromb. Haemost. in press.

60. Hirosawa S., Fahner J.B., Salier J.P., Wu C.T., Lovrien E.W. and Kurachi K. (1990) Proc. Natl.Acad.Sci.USA 87: 4421-4425.

61. Hougie C. and Twomey J.J. (1967) Lancet i: 699.

62. Huang M.N., Kasper C.K., Roberts H.R, et al (1989) Blood 73: 718-721

63. Jagadeeswaran P., Dept of Cellular \& Structural Biology, Univ. of Texas, San Antonio, Texas, USA, unpublished. 
64. Ketterling R.P., Bottema C.D.K., Koeberl D.D., Ii, S. and Sommer, S.S. (1991a) Hum.Genet. 87: 333-337.

65. Ketterling R.P., Bottema C.D.K., Phillips J.A. and Sommer S.S. (1991b) Genomics 10: 1093-1096.

66. Ketterling R.P., Vielhaber E., Bottema C.D.K., Schaid D.J., Phillips J.A., III, Sexauer C.L. and Sommer S.S. (1993) Am J. Hum. Genet. in press.

67. Knobloch O. and Zoll B., Dept. of Human Genetics, Univ. of Göttinggen, Goßlerstr. 12d, W-3400 Göttingen, FRG, unpublished.

68. Koeberl D.D., Bottema C.D.K., Buerstedde J.M., and Sommer S.S. (1989) Am. J. Hum. Genetics 45: 448-457.

69. Koeberl D.D., Bottema C.D.K., Ketterling R.P., Bridge P.J., Lillicrap D.P., and Sommer S.S. (1990a) Am.J.Hum.Genet. 47: 202-217..

70. Koeberl D.D., Bottema C.D.K., Sarkar G., Ketterling R.P., Chen S.H. and Sommer S.S. (1990b) Hum.Genet. 84: 387-390..

71. Kuze K., Nishimura T., Naka H., Morimoto H., Kuwahara I., Morichika S., Mikami S., Yoshioka A., Matsui A. and Fukui H.(1992) Inter. J. Haematol. 55 Suppl. 1: 272(abs).

72. Liddell M.B., Lillicrap D.P., Peake I.R. and Bloom A.L. (1989a) Br.J.Haematol. 72: 208-215.

73. Liddell M.B., Peake I.R., Taylor S.A.M. et al (1989b) Br.J.Haematol 72: $556-560$.

74. Lillicrap D. and Windsor S., DNA Diagnostic Laboratory, Kingston General Hospital, Kingston, Ontario, unpublished.

75. Lillicrap D., Windsor S. et al, Dept. Pathology, Queen's University, Kingston, Ont. Canada, unpublished.

76. Lin S.-W. and Shen M.C. (1991) Thromb. Haemost. 66: 459-463.

77. Lin S.-W. et al (1993) Thromb. Haemost., in press.

78. Lin S.-W. et al., Inst. Med. Techn. Coll. of Medicine, National Taiwan Univ. 1 Chang-te St., Taipei, Taiwan 10016, Rep. China, unpublished.

79. Ljung R. et al, Dept. Coagulation Disorders, Univ. Lund, Malmö General Hospital, Malmö, Sweden, unpublished.

80. Lozier J.N., Monroe D.M., Stanfield-Oakley S.A. et al (1990) Blood 75: 1097-1104.

81. Ludwig M., Schwaab R., Olek K., Brackmann H.H., Egli H. (1988) Thromb. Haemost. 59: 340 .

82. Ludwig M., Schwaab R., Eigel A., Horst J., Egli H., Brackmann H-H and Olek K. (1989) Am.J.Hum.Genet. 45: 115-122.

83. Ludwig M., Brackmann H.H. and Olek K. (1991) Klin.Wochenschr. 69: 196-200.

84. Ludwig M., Grimm T., Brackmann H.H., Olek K. (1992a) Am. J. Hum. Genet. 50: 164-173.

85. Ludwig M., Sabharwal A.K., Brackmann H.H., Olek K., Smith K.J., Birktoft J.J. and Bajaj S.P. (1992b) Blood 79: 1225-1232.

86. Ludwig M. et al, Dept. Clinical Biochemistry, Univ. of Bonn, SigmundFreud-Str. 25, W-5300 Bonn 1, FRG, unpublished.

87. Maekawa H., Sugo T., Muramatsu S. et al (1991) Jpn. J. Thromb. Haemost. 2: 400 (abstract).

88. Matsushita T., Tanimoto M., Yamamoto K., Sugiura I., Hamaguchi M., Takamatsu J., Kamiya T. and Saito H. (1990) J.Lab.Clin.Med. 116: $492-497$.

89. Miyata T., Sakai T., Sugimoto M., Naka H., Yamamoto K., Yoshioka A., Fukui H., Mitsui K., Kamiya K., Umeyama H. and Iwanaga S. (1991) Biochemistry 30: 11286-11291.

90. Monroe D.M., McCord D.M., Huang M.N. et al (1989) Blood 73 $1540-1544$

91. Montandon, A.J., Green, P.M., Giannelli, F. and Bentley, D.R. (1989) Nucl. Acids Res. 17: 3347-3358.

92. Montandon, A.J., Green, P.M., Bentley, D.R., Ljung, R., Nilsson, I.M. and Giannelli, F. (1990a) Hum. Gen. 85: 200-204.

93. Montandon A.J., Makris M., Green P.M., Coffey A.J., Preston F.E. and Giannelli F. (1990b) Br.J.Haematol. 76: suppl.1 p.8.

94. Nishimura T., Naka H., Kuze K. et al (1990) Acta Haematol. Jpn. 53: $1030-1035$.

95. Nishimura H., Takeya H., Suehiro K., Okamura T., Murakawa M., Niho Y., Miyata T. and Iwanaga S. (1991) Thromb. Haemost. 65: 712 (abstract).

96. Nishimura H., Takao T., Hase S., Shimonishi Y. \& Iwanaga S. (1992) J. Biol. Chem. 267: 17520-17525.

97. Noyes C.M., Griffith M.J., Roberts H.R., and Lundblad R.L. (1983) Proc. Natl. Acad. Sci. (USA) 80: 4200-4202.

98. Pang C.P., Crossley M., Kent G. and Brownlee G.G. (1990) Nucl.Acids Res. 18: 6731-6732.

99. Picketts et al, Department of Pathology, Queen's University, Kingston, Ontario, Canada, unpublished.

100. Picketts D.J., Bridge P.J. and Lillicrap D. (1990) Blood 76 (Suppl.1): 1721a (abstract).

101. Poon M.-C., Chui D.H.K., Patterson M., Starozik D.M., Dimnik L.S. and Hoar D.I. (1987) J.Clin.Invest. 79: 1204-1209.
102. Poon M.-C., Anand S., Fraser B.M., Hoar B.I., Sinclair D.G.D. (1993) J. Lab. Clin. Med. in press.

103. Poort S.R., Briët E., Bertina R.M. and Reitsma P.H. (1989a) Nucl. Acids Res. 17: 3614.

104. Poort S.R., Briët E., Bertina R.M. and Reitsma P.H. (1989b) Nucl. Acids. Res. 17: 5869.

105. Poort S.R., Briët E., Bertina R.M. and Reitsma P.H. (1990) Thromb.Haemost. 64: 379-384.

106. Poort S.R. et al, Hemostasis \& Thrombosis Research Unit, 2300 RC Leiden, The Netherlands, unpublished.

107. Rao K.J., Lyman G., Hamsabhushami K., Scott, J.P. \& Jagadeeswaran, P. (1990) Molecular \& Cellular Probes 4: 335-340.

108. Rees D.J.G., Rizza C.R. and Brownlee G.G. (1985) Nature 316: 643-645.

109. Reitsma P.H., Bertina R.M., Ploos van Amstel J.K., Riemens A. and Briët E. (1988) Blood 72: 1074-1076.

110. Reitsma P.H., Mandalaki T, Kasper C.K. and Briët E. (1989) Blood 73: $743-746$.

111. Reitsma P.H. et al, Hemostasis \& Thrombosis Research Unit, 2300 RC Leiden, The Netherlands, unpublished.

112. Ritchie D.B.C., Tam B.M. and MacGillivray R.T.A. (1989) Alberta Heritage Foundation for Medical Research Heritage Days. Abstract.

113. Ritchie D.B.C., Tam B.M. and MacGillivray R.T.A. Dept. Biochem. Univ. Alberta, Edmonton, Alberta, Canada, unpublished.

114. Rose V. and High K.A. Unpublished observation, Depts. of Medicine and Pathology, Univ. of North Carolina at Chapel Hill, Chapel Hill, NC.

115. Royle G., Van de Water N.S., Berry E., Ockelford P.A. and Browett P.J. (1990) Brit.J.Haematol. 77: $191-194$.

116. Saad S., Rowley G., Green P.M. \& Giannelli F. Paedeatric Research Unit, Guy's Hospital Tower, London, UK, unpublished.

117. Sakai T., Yoshioka A., Yamamoto K., Niinomi K., Fujimura Y., Fukui H., Miyata T. and Iwanaga S. (1989) J.Biochem. 105: 756-759.

118. Schach B.G., Yoshitake S., Davie E.W. (1987) J. Clin. Invest. 80: 1023-1028.

119. Siguret V., Amselem S., Vidaud M. et al (1988) Br.J.Haematol. 70: 411-416.

120. Solera J., Magallón M., Martín-Villar J. and Coloma A. (1991) Br. J. Haematol. 78: 385-389.

121. Solera J. et al Dept. Biochem. Univ. Autonoma, Madrid, 28029, Spain, unpublished.

122. Spitzer S.G., Pendurthi U.R., Kasper C.K., and Bajaj S.P. (1988) J. Biol. Chem. 263: 10,545-10,548.

123. Spitzer S.G., Warn-Cramer B.J., Kasper C.K., and Bajaj S.P. (1990a) Biochem. J. 265: 219-225.

124. Spitzer S.G., Kuppuswamy, M.N., Saini R., Kasper C.K., Birktoft J.J. and Bajaj S.P. (1990b) Blood 76: 1530-1537.

125. Suehiro K., Kawabata S., Miyata T., Takeya H., Takamatsu J., Ogata K. Kamiya T., Saito H., Niho Y. and Iwanaga S. (1989) J.Biol.Chem. 264: 21257-21265.

126. Suehiro K., Miyata T., Takeya H., Takamatsu J., Saito H., Murakawa M., Okamura T., Niho Y. and Iwanaga S. (1990) Thromb. Res. 60: 311-320.

127. Sugimoto M., Miyata T., Kawabata S., Yoshioka A., Fukui H., Takahashi H. and Iwanaga S. (1988) J. Biochem. 104: 878-880.

128. Sugimoto M., Miyata T., Kawabata S., Yoshioka A., Fukui H. and Iwanaga S. (1989) Br.J.Haematol. 72: 216-221.

129. Tam B.M., MacGillivray R.T.A. and Ritchie D.B.C. (1991) Thromb. Haemost. 65: 968 (abstract).

130. Tarnower A. and Smith K., (1991) Thromb. Haemost. 65: 967 (abstract).

131. Taylor S.A.M. (1990). Ph.D. Thesis, Queen's University, Kingston, Ontario.

132. Taylor S.A.M., Liddell M.B., Peake I.R., Bloom A.L. and Lillicrap D.P. (1990) Br. J. Haematol. 75: 217-221..

133. Taylor S.A.M., Deugau K.V. and Lillicrap D.P. (1991) Proc.Natl.Acad.Sci.USA 88: 39-42.

134. Thompson A.R. (1990) Prog.Hemost.Thromb. 10: 175-214.

135. Thompson A.R., Chen S.H., and Brayer G.D. (1989) Blood 74 (suppl): 134a (abstract).

136. Thompson A.R. and Chen S.H. (1992) Circulation I: 686.

137. Thompson A.R. and Chen S.H. (1993) Methods in Enzymology (Lorand \& Mann, guest editors), in press.

138. Thompson A.R., Bajaj S.P., Chen S.H. and MacGillivray R.T.A. (1990) Lancet $\mathrm{i}: 418$.

139. Thompson A.R., Schoof J.M., Weinmann A.F. and Chen S.H. (1992a) Thromb. Res. 65: 289-295.

140. Thompson A.R., Brayer G.D. and Chen S.H. (1992b) Blood 80: 367a.

141. Toomey J.R., Stafford D., and Smith K. (1988) Blood 72 (suppl): 312a (abstract). 
142. Tsang T.C., Bentley D.R., Mibashan R.S. and Giannelli F. (1988) EMBO J. 7: 3009-3015

143. Van de Water N.S., Ockelford P.A., Berry E.W., Browett P.J., School of Medicine, Univ. Auckland, New Zealand, unpublished.

144. Vidaud M., Attree O., Schaad O., Vidaud D., Edelstein S. and Goossens M. (1988) Blood 72 Suppl.1, Abstract 1161.

145. Vidaud M. (1990) Thesis, Paris VII University.

146. Wang N.S., Chen S.H. and Thompson A.R. (1990a) Thromb. Haemost. 64: 302-306.

147. Wang N.S., Zhang N.S., Thompson A.R., and Chen S.H. (1990b) Thromb. Haemost. 63: 24-26.
148. Ware J., Davis L., Frazier D. et al (1988) Blood 72: 820-822.

149. Ware J., Diuguid D.L., Liebman H.A. et al (1989) J. Biol. Chem. 264: $11,401-11,406$

150. Winship P.R. (1986) D.Phil thesis, Oxford University.

151. Winship P.R. (1990) Nucl. Acids Res. 18: 1310.

152. Winship P.R. and Dragon A.C. (1991) Br.J.Haematol. 77: 102-109.

153. Wulf K., Hermann F.H., et al, Dept. Med. Genetics, Univ. of Greifswald, Fleischmannstr. 42/44, O-2200, Greifswald, FRG, unpublished.

154. Yoshitake S., Schach B.G., Foster D.C., Davie E.W. and Kurachi K. (1985) Biochemistry 24: 3736-3750.

Characterized Point Mutations and Short Deletions/Additions in patients with Haemophilia B (Christmas disease)

\begin{tabular}{|c|c|c|c|c|c|c|c|}
\hline Patient $^{7}$ & $\begin{array}{l}\text { Clotting } \\
\text { (normal } \\
=100 \% \text { ) }\end{array}$ & $\begin{array}{l}\text { Antigen } \\
\text { (normal } \\
=100 \% \text { ) }\end{array}$ & $\begin{array}{l}\text { Nucleotide }^{1,3,8} \\
\text { position \& } \\
\text { mutation }\end{array}$ & $\begin{array}{l}\text { Amino acid } \\
\text { change }\end{array}$ & Comments $^{3}$ & Reference & $\begin{array}{l}\text { Patient } \\
\text { Identity } \\
\text { Number }^{10}\end{array}$ \\
\hline HB5, Japan & $<1$ & $<1$ & $-793, G \rightarrow A$ & None & Double (see 20,551), N? & Matsushita et al (1990) & 1 \\
\hline Brandenburg & $<1$ & $<1$ & $-26, G \rightarrow C$ & None & $\begin{array}{l}\text { Promoter, LF-A1/HNF4- } \\
\text { binding site, ARE }\end{array}$ & $\begin{array}{l}\text { Ludwig et al } \\
\text { Crossley et al (1992) }\end{array}$ & 395 \\
\hline Leyden 1 & $<1 \rightarrow 60^{5}$ & $<1 \rightarrow 60$ & $-20, T \rightarrow A$ & None & $\begin{array}{l}\text { Dinding site, AKIE } \\
\text { Promoter }\end{array}$ & Reitsma et al (1988) & 2 \\
\hline Datteln & $<1 \rightarrow 36^{5}$ & $<1 \rightarrow 36^{5}$ & $-20, T \rightarrow A$ & None & $\begin{array}{l}\text { Promoter,LF-A1/HNF4- } \\
\text { binding site }\end{array}$ & Ludwig et al & 3 \\
\hline Marseille & $7 \rightarrow 20^{5}$ & & $-20, T \rightarrow C$ & None & $\begin{array}{l}\text { Promoter, de novo in } \\
\text { MGF }\end{array}$ & Ghanem et al (1993) & 396 \\
\hline High Wycombe & $13 \rightarrow 70^{5}$ & & $-6, G \rightarrow A^{4}$ & None & Promoter & Crossley et al (1990) & 4 \\
\hline Leyden, USA1 & 10 & 13 & $-6, G \rightarrow A^{4}$ & None & Promoter & Hirosawa et al (1990) & 5 \\
\hline Calgary 18 & & $-6, G \rightarrow A^{4}$ & None & Promoter & Poon et al (1993) & 577 & \\
\hline Rodez & $1 \rightarrow$ norm $^{5}$ & & $-6, G \rightarrow A^{4}$ & None & Promoter & Ghanem et al (1993) & 578 \\
\hline Toulouse & $1 \rightarrow 30^{5}$ & $1 \rightarrow 30^{5}$ & $-6, G \rightarrow C$ & None & Promoter & Gispert et al (1989) & 6 \\
\hline Toronto 20 & 3 & & $-5, A \rightarrow T$ & None & Promoter & Picketts et al & 7 \\
\hline UK 110 & 15 & & $-5, A \rightarrow G$ & None & Promoter & Saad, Rowley et al & 579 \\
\hline UK 161 & 7 & & $-5, A \rightarrow G$ & None & Promoter & Saad, Rowley et al & 580 \\
\hline Bangkok & 5 & & 6,7 or $8, \Delta 1$ & None & Promoter & Hall et al (1992) & 581 \\
\hline Leyden, USA2 & $<2 \rightarrow 37^{5}$ & & $6, T \rightarrow A$ & None & Promoter & Freedenberg, Black (1991) & 397 \\
\hline Leyden, NZ & $1 \rightarrow 32^{5}$ & & $8, T \rightarrow C$ & None & $\begin{array}{l}\text { Promoter, C/EBP binding } \\
\text { site, de novo in mother }\end{array}$ & Royle et al (1990) & 8 \\
\hline Leyden $2<1 \rightarrow 60^{5}$ & $<1 \rightarrow 60^{5}$ & $13, A \rightarrow G$ & None & Promoter & Reitsma et al (1989) & 9 & \\
\hline HB13 & 32 & & $13, A \rightarrow G$ & None & Promoter & Koeberl et al (1989) & 10 \\
\hline Norwich & $3 \rightarrow 35^{5}$ & & $13, A \rightarrow G$ & None & Promoter & Crossley et al (1989) & 11 \\
\hline Poitiers & 12 -norm 5 & & $13, A \rightarrow G$ & None & Promoter & Ghanem et al (1993) & 398 \\
\hline Leyden 3 & $<1 \rightarrow 60^{5}$ & $<1 \rightarrow 60^{5}$ & $13, \Delta 1$ & None & Promoter & Reitsma et al (1989) & 12 \\
\hline Aachen & $<1$ & & $13, A \rightarrow C$ & None & $\begin{array}{l}\text { Promoter, } \\
\text { C/EBP-binding site }\end{array}$ & Ludwig et al & 13 \\
\hline Riegelsberg & $<1$ & $<1$ & $37, G \rightarrow A^{4}$ & $-44, R \rightarrow H$ & Double (see 31,084$), \mathrm{N}$ & Ludwig et al (1992a) & 14 \\
\hline Calgary 4 & $<1$ & $<1$ & $48, A \rightarrow T$ & $-40, I \rightarrow F$ & Double (see 31,133 ), N & Poon et al (1993) & 399 \\
\hline UK 22 & 2 & $<1$ & $79, \mathrm{~T} \rightarrow \mathrm{A}$ & $-30, I \rightarrow N$ & Signal peptide & Green et al (1992b) & 15 \\
\hline Toronto 23 & $<1$ & & $89, \Delta 1$ & -27 & Frameshift & Lillicrap \& Windsor & 582 \\
\hline HB130 & 20 & & $111, T \rightarrow C$ & $-19, C \rightarrow R$ & Signal peptide & Bottema et al (1991b) & 400 \\
\hline UK 36 & & $<1$ & $111-120, \Delta 10$ & -19 & $\begin{array}{l}\text { Frameshift, } \\
\text { Donor splice (a) }\end{array}$ & Green, Montandon et al & 16 \\
\hline Meaux & $<1$ & & $112, \Delta 1$ & -19 & Frameshift & Ghanem et al (1993) & 401 \\
\hline Recklinghausen & $<1$ & $<1$ & 114 , Ins AT & -18 & Frameshift & Ludwig et al & 17 \\
\hline HB64 & $<1$ & 2 & $117, \mathrm{G} \rightarrow \mathrm{A}$ & $-17, V \rightarrow I$ & Donor splice (a)? & Bottema et al (1991a) & 402 \\
\hline Malmö 33 & 3 & 2 & $122, \mathrm{G} \rightarrow \mathrm{A}$ & & $\begin{array}{l}\text { Donor splice (a), de novo } \\
\text { in MGF }\end{array}$ & Green et al (1991b) & 18 \\
\hline HB135 & & & $6,320, T \rightarrow G$ & & Acceptor splice (b) & Bottema et al (1991b) & 403 \\
\hline UK 70 & $<1$ & & $6,325, G \rightarrow A$ & & Acceptor splice (b) & Saad, Rowley et al & 404 \\
\hline Spain & $<1$ & & $6,325, \mathrm{G} \rightarrow \mathrm{T}$ & & Acceptor splice (b) & Ghanem et al (1993) & 405 \\
\hline (footnote 11) & $1.8^{11}$ & $34^{11}$ & $6,364, C \rightarrow T^{4}$ & $-4, R \rightarrow W$ & 16 cases & $\begin{array}{l}\text { Ref } N^{o s}:(12,43,47,48,53 \text {, } \\
65,77,84,108) \\
\text { PINs: }(19-28,406-8 \text {, } \\
583-5)\end{array}$ & \\
\hline (footnote 11) & $<1^{11}$ & $43^{11}$ & $6,365, \mathrm{G} \rightarrow \mathrm{T}$ & $-4, R \rightarrow L$ & 6 cases & $\begin{array}{l}\text { Ref Nos: }(43,67,84) \\
\text { PINs: }(30-32,409,410)\end{array}$ & \\
\hline (footnote 11) & $<1^{11}$ & $57^{11}$ & $6,365, G \rightarrow A^{4}$ & $-4, R \rightarrow Q$ & $\begin{array}{l}29 \text { cases. Abnormal } \\
\text { carboxylation, circulates } \\
\text { with propeptide }\end{array}$ & $\begin{array}{l}\text { Ref Nos: }(5,9,13,17,22,30 \text {, } \\
43,47,57,67,70,74,75, \\
84,99,113,125, \\
136,140,146) \\
\text { PINs: }(33-45,411-8, \\
\text { 586-94) }\end{array}$ & \\
\hline Seattle E & $<1$ & 37 & $6,372, G \rightarrow T$ & $-2, \mathrm{~K} \rightarrow \mathrm{N}$ & & Thompson et al (1992a) & 419 \\
\hline Cambridge & $<1$ & 80 & $6,375, \mathrm{G} \rightarrow \mathrm{C} / \mathrm{T}^{2}$ & $-1, \mathrm{R} \rightarrow \mathrm{S}$ & $\begin{array}{l}\text { Abnormal carboxylation, } \\
\text { circulates with propeptide }\end{array}$ & Diuguid et al (1986) & 46 \\
\hline
\end{tabular}


3080 Nucleic Acids Research, 1993, Vol. 21, No. 13

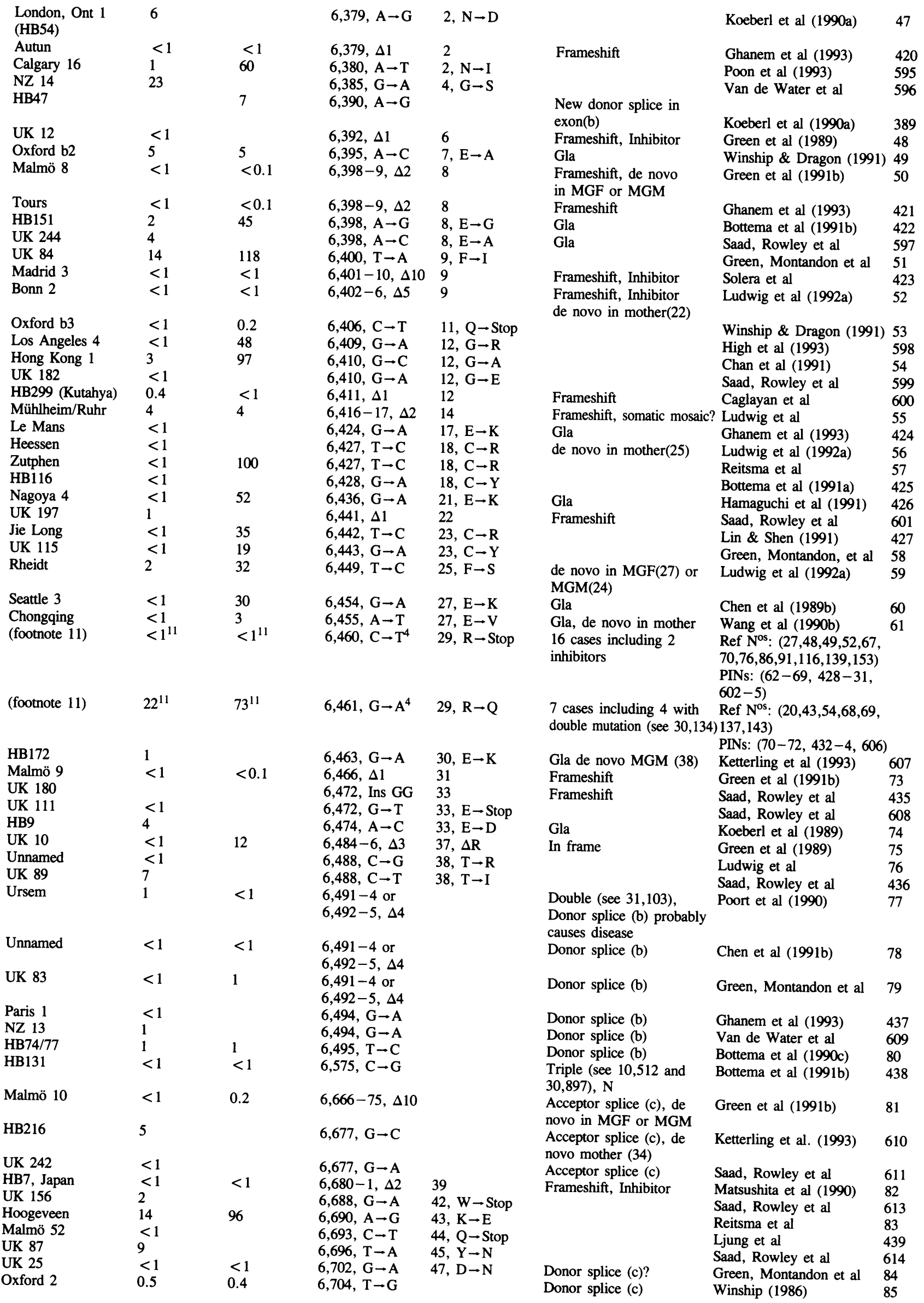




\begin{tabular}{|c|c|c|c|c|c|c|c|}
\hline Pirmasens & $<1$ & $<1$ & $6,704, T \rightarrow C$ & & Donor splice (c) & Ludwig et al & 86 \\
\hline Nantes & 3,13 & & $6,704, T \rightarrow C$ & & $\begin{array}{l}\text { Donor splice }(\mathrm{c}) \text {, female } \\
\text { to female transmission }\end{array}$ & Ghanem et al (1993) & 440 \\
\hline HB20 (Bogazici) & 5 & & $6,707, G \rightarrow C$ & & Donor splice (c) & Caglayan et al & 615 \\
\hline UK 128 & 1,3 & & $6,707, G \rightarrow A$ & & Donor splice (c) & Saad, Rowley et al & 616 \\
\hline HB197 & $<1$ & 27 & $10,389, A \rightarrow G$ & & $\begin{array}{l}\text { Acceptor splice (d), de } \\
\text { novo MGF (27) }\end{array}$ & Ketterling et al. (1993) & 617 \\
\hline Toronto 16 (HB53) & 3 & & $10,391, \mathrm{G} \rightarrow \mathrm{A}$ & & Acceptor splice (d) & Koeberl et al (1990a) & 87 \\
\hline Alabama & 10 & 100 & $10,392, A \rightarrow G$ & $47, D \rightarrow G$ & & Davis et al (1987) & 88 \\
\hline HB75 & 14 & 80 & $10,393, T \rightarrow A$ & $47, D \rightarrow E$ & & Bottema et al $(1990 \mathrm{c})$ & 89 \\
\hline Oxford d3 & 1 & 90 & $10,393, T \rightarrow A$ & $47, \mathrm{D} \rightarrow \mathrm{E}$ & & Winship \& Dragon (1991) & 90 \\
\hline Tainan & 28 & 101 & $10,394, G \rightarrow A$ & $48, G \rightarrow R$ & & Lin \& Shen (1991) & 441 \\
\hline Malmö 27 & 19 & 108 & $10,395, G \rightarrow T$ & $48, G \rightarrow V$ & de novo in mother & Green et al (1991b) & 91 \\
\hline UK 86 & & & $10,397-9, \Delta 3$, Ins $A$ & 49 & Frameshift & Green, Montandon et al & 92 \\
\hline Clermont & $<1$ & & $10,400, C \rightarrow T$ & 50, Q $\rightarrow$ Stop & & Costes et al & 618 \\
\hline New London & $<1$ & 114 & $10,401, A \rightarrow C$ & $50, \mathrm{Q} \rightarrow \mathrm{P}$ & Decreased XIa activation & Lozier et al (1990) & 93 \\
\hline UK 175 & 30 & & $10,405, T \rightarrow G$ & $51, C \rightarrow W$ & Female carrier & Saad, Rowley et al & 619 \\
\hline Lhuis & $<1$ & $<1$ & $10,405, T \rightarrow G$ & $51, C \rightarrow W$ & & Ghanem et al (1993) & 620 \\
\hline HB97 & $<1$ & 20 & $10,406, \mathrm{G} \rightarrow \mathrm{T}$ & $52, \mathrm{E} \rightarrow$ Stop & & Bottema et al (1991a) & 442 \\
\hline GER 5 & $<1$ & & $10,406, G \rightarrow T$ & $52, \mathrm{E} \rightarrow$ Stop & de novo in mother (23) & Knobloch \& Zoll & 621 \\
\hline Hollywood & 11 & 58 & $10,415, C \rightarrow G$ & $55, \mathrm{P} \rightarrow \mathrm{A}$ & & Spitzer et al (1990b) & 94 \\
\hline UK 7 & $10-12$ & 49 & $10,415, C \rightarrow G$ & $55, \mathrm{P} \rightarrow \mathrm{A}$ & & Green et al (1989) & 95 \\
\hline Unnamed & 10 & $<30$ & $10,415, C \rightarrow G$ & $55, P \rightarrow A$ & & Chen et al (1991a) & 96 \\
\hline Malmö 21 & 12 & 52 & $10,415, C \rightarrow T$ & $55, \mathrm{P} \rightarrow \mathrm{S}$ & & Green et al (1991b) & 97 \\
\hline Malmö 22 & & 26 & $10,416, C \rightarrow T$ & $55, \mathrm{P} \rightarrow \mathrm{L}$ & & Green et al (1991b) & 98 \\
\hline HB180 & 6 & 34 & $10,416, C \rightarrow A$ & $55, \mathrm{P} \rightarrow \mathrm{Q}$ & de novo MGF (38) & Ketterling et al. (1993) & 622 \\
\hline Basel & $<1$ & & $10,418, T \rightarrow C$ & $56, C \rightarrow R$ & & Alkan et al (1991) & 99 \\
\hline Kleve & $<1$ & $<1$ & $10,419, G \rightarrow C$ & $56, C \rightarrow S$ & & Ludwig et al (1991) & 100 \\
\hline Toronto 2 (HB32) & 1 & 2 & $10,419, G \rightarrow A$ & $56, C \rightarrow Y$ & & Koeberl et al (1990a) & 101 \\
\hline UK 160 & 18 & & $10,427, \mathrm{G} \rightarrow \mathrm{A}$ & $59, \mathrm{G} \rightarrow \mathrm{S}$ & & Saad, Rowley et al & 443 \\
\hline HB141 & 2 & & $10,428, \mathrm{G} \rightarrow \mathrm{T}$ & $59, \mathrm{G} \rightarrow \mathrm{V}$ & De novo in mother(40) & Bottema et al (1991b) & 444 \\
\hline (footnote 11) & $13^{11}$ & $29^{11}$ & $10,430, G \rightarrow A^{4}$ & $60, G \rightarrow S$ & 29 cases & $\begin{array}{l}\text { Ketterling et al }(1993) \\
\text { Ref } N^{\text {os: }}(19-21,32,49,65 \\
68,69,102,104,106,136, \\
139,152) \\
\text { PINs: }(102-12,390-1 \text {, } \\
445-57,624-6)\end{array}$ & \\
\hline HB154 & $<1$ & 6 & $10,430, G \rightarrow C$ & $60, G \rightarrow R$ & & Bottema et al (1991b) & 458 \\
\hline Toronto 6 (HB37) & 1 & 2 & $10,431, G \rightarrow A$ & $60, G \rightarrow D$ & & Koeberl et al (1990a) & 113 \\
\hline UK 176 & & & $10,437, G \rightarrow A$ & $62, \mathrm{C} \rightarrow \mathrm{Y}$ & & Saad, Rowley et al & 627 \\
\hline Oxford d1 & 3 & 117 & $10,442, G \rightarrow A$ & $64, \mathrm{D} \rightarrow \mathrm{N}$ & $\beta$ Hydroxyaspartate & Winship \& Dragon (1991) & 114 \\
\hline UK 6 & 8 & 87 & $10,443, A \rightarrow G$ & $64, D \rightarrow G$ & $\beta$ Hydroxyaspartate & Green et al (1989) & 115 \\
\hline Madrid 4 & 5 & & $10,449, T \rightarrow C$ & $66, I \rightarrow T$ & & Damas et al & 628 \\
\hline Rouen & 30 & & $10,449, T \rightarrow C$ & $66, I \rightarrow T$ & & Ghanem et al (1993) & 629 \\
\hline Trier & $<1$ & & $10,458, A \rightarrow G$ & $69, Y \rightarrow C$ & $\begin{array}{l}\text { Haplotype differs from } \\
\text { GER } 6\end{array}$ & Ludwig et al & 116 \\
\hline GER 6 & $<1$ & & $10,458, A \rightarrow G$ & $69, \mathrm{Y} \rightarrow \mathrm{C}$ & $\begin{array}{l}\text { de novo in mother (33), } \\
\text { Haplotype differs from } 116\end{array}$ & 6 & 630 \\
\hline Murten & $<1$ & & $10,458, A \rightarrow G$ & $69, Y \rightarrow C$ & & Ghanem et al (1993) & 631 \\
\hline Almere & & & $10,463, T \rightarrow A$ & $71, \mathrm{C} \rightarrow \mathrm{S}$ & & Deutz et al & 632 \\
\hline UK 167 & & & $10,464, G \rightarrow C$ & $71, \mathrm{C} \rightarrow \mathrm{S}$ & & Saad, Rowley et al & 633 \\
\hline UK 200 & 6 & & $10,466, T \rightarrow A$ & $72, \mathrm{~W} \rightarrow \mathrm{R}$ & & Saad, Rowley et al & 634 \\
\hline UK 150 & & & $10,466, T \rightarrow A$ & $72, \mathrm{~W} \rightarrow \mathrm{R}$ & & Saad, Rowley et al & 635 \\
\hline Fort de France & $<1$ & & $10,468, G \rightarrow A$ & $72, \mathrm{~W} \rightarrow$ Stop & & Ghanem et al (1993) & 636 \\
\hline GER 7 & $<1$ & & $10,470, G \rightarrow A$ & $73, \mathrm{C} \rightarrow \mathrm{Y}$ & & Knobloch \& Zoll & 637 \\
\hline Paris 3 & $<1$ & & $10,471, T \rightarrow A$ & 73, $\mathrm{C} \rightarrow$ Stop & & Ghanem et al (1993) & 638 \\
\hline UK 67 & 6 & 12 & $10,479, \mathrm{G} \rightarrow \mathrm{T}$ & $76, \mathrm{G} \rightarrow \mathrm{V}$ & Female carrier & Green, Montandon et al & 117 \\
\hline UK 19 & & & $10,482, T \rightarrow G$ & $77, \mathrm{~F} \rightarrow \mathrm{Y}$ & & Green, Montandon et al & 118 \\
\hline UK 132 & & & $10,507-10, \Delta 4$ & & $\begin{array}{l}\text { Donor splice }(\mathrm{d}) \text {, } \\
\text { normal carrier female }\end{array}$ & Green, Montandon et al & 119 \\
\hline Toronto 8 (HB39) & 2 & 3 & $10,512, A \rightarrow G$ & & Double (see 30,864),N? & Koeberl et al (1990a) & 120 \\
\hline Paris 5 & $<1$ & & $10,512, A \rightarrow G$ & & Double (see 17,799),N? & Ghanem et al (1993) & 639 \\
\hline HB131 & $<1$ & $<1$ & $10,512, A \rightarrow G$ & & $\begin{array}{l}\text { Triple (see } 6575 \text { \& } \\
\text { 30,897), N? }\end{array}$ & Bottema et al (1991b) & 438 \\
\hline HB6 & 20 & & $17,660-3, \Delta 4$ & & Acceptor splice (e) & Koeberl et al (1989) & 121 \\
\hline Toronto 14 (HB48) & 3 & 3 & $17,667, A \rightarrow G$ & & Acceptor splice (e) & Koeberl et al (1990a) & 122 \\
\hline Malmö 11 & $<1$ & $<1$ & $17,668, G \rightarrow C$ & & $\begin{array}{l}\text { Acceptor splice (e), de } \\
\text { novo in MGF }\end{array}$ & Green et al (1991b) & 123 \\
\hline Seattle 2 & $<1$ & $<1$ & $17,669, \Delta 1$ & 85 & Frameshift & Schach et al (1987) & 124 \\
\hline HB147 & 2 & 2 & $17,669-75 \Delta 7$ & 85 & $\begin{array}{l}\text { Frameshift de novo } \\
\text { mother (33) }\end{array}$ & Ketterling et al. (1993) & 640 \\
\hline UK 98 & $<2$ & & $17,677, T \rightarrow C$ & $88, C \rightarrow R$ & & Saad, Rowley et al & 641 \\
\hline Königswinter & $<1$ & & $17,678, G \rightarrow C$ & $88, C \rightarrow S$ & & Ludwig et al & 125 \\
\hline UK 125 & 15 & & $17,684, T \rightarrow C$ & $90, I \rightarrow T$ & & Saad, Rowley et al & 642 \\
\hline Fukuoka & 2 & 66 & $17,689, A \rightarrow C^{2}$ & $92, \mathrm{~N} \rightarrow \mathrm{H}$ & & Nishimura et al (1991) & 126 \\
\hline Chelles & $<1$ & & $17,691, T \rightarrow A$ & $92, \mathrm{~N} \rightarrow \mathrm{K}$ & & Ghanem et al (1993) & 459 \\
\hline
\end{tabular}


3082 Nucleic Acids Research, 1993, Vol. 21, No. 13

\begin{tabular}{|c|c|c|c|c|c|c|c|}
\hline Amiens & $<1$ & & $17,691, T \rightarrow A$ & $92, \mathrm{~N} \rightarrow \mathrm{K}$ & & Ghanem et al (1993) & 460 \\
\hline HB256 & 3 & 22 & $17,692, \mathrm{G} \rightarrow \mathrm{A}$ & $93, G \rightarrow S$ & de novo mother (27) & Ketterling et al. (1993) & 643 \\
\hline HB106 & 1 & 84 & $17,697, \mathrm{~A} \rightarrow \mathrm{T}$ & $94, \mathrm{R} \rightarrow \mathrm{S}$ & & Bottema et al (1991a) & 461 \\
\hline HB134 & 5 & & $17,699, \mathrm{G} \rightarrow \mathrm{A}$ & $95, C \rightarrow Y$ & & Bottema et al (1991b) & 462 \\
\hline HB157 & $<1$ & 6 & $17,699, \mathrm{G} \rightarrow \mathrm{A}$ & $95, \mathrm{C}-\mathrm{Y}$ & Haplotype differs from 462 & Bottema et al (1991b) & 463 \\
\hline Edmonton 1 & $<1$ & & $17,700, C \rightarrow A$ & 95, C $\rightarrow$ Stop & & Tam et al (1991) & 127 \\
\hline Malmö 51 & $<1$ & & $17,700, C \rightarrow G$ & $95, \mathrm{C} \rightarrow \mathrm{W}$ & & Ljung et al & 464 \\
\hline GER 8 & & & $17,705, A \rightarrow C$ & $97, Q \rightarrow P$ & & Knobloch \& Zoll & 644 \\
\hline Taichung & $<1$ & $<13$ & $17,706, \Delta 1$ & 97 & Frameshift & Lin et al (1993) & 645 \\
\hline Hamilton 1 (HB45) & $<1$ & & $17,710, T \rightarrow C$ & 99, C $\rightarrow R$ & & Koeberl et al (1990a) & 128 \\
\hline UK 243 & $<1$ & & 17,717, Ins A & 101 & Frameshift & Saad, Rowley et al & 646 \\
\hline NZ 4 & $<1$ & & 17,718 , Ins A & 101 & Frameshift & Van de Water et al & 465 \\
\hline UK 50 & $<1$ & $<1$ & 17,727, Ins TT & 105 & Frameshift & Green, Montandon et al & 129 \\
\hline Malmö 35 & 21 & 14 & $17,736, \mathrm{G} \rightarrow \mathrm{A}$ & 107 , None & cryptic splice? & Green et al (1991b) & 130 \\
\hline Malmö 42 & 20 & 24 & $17,736, G \rightarrow A$ & 107 , None & cryptic splice? & Green et al (1991b) & 131 \\
\hline Malmö 37 & 15 & 24 & $17,736, G \rightarrow A$ & 107, None & cryptic splice? & Green et al (1991b) & 132 \\
\hline Malmö 55 & 18 & & $17,736, \mathbf{G} \rightarrow \mathrm{A}$ & 107 , None & cryptic splice? & Ljung et al & 647 \\
\hline Malmö 56 & 17 & & $17,736, \mathbf{G} \rightarrow \mathbf{A}$ & 107 , None & cryptic splice? & Ljung et al & 648 \\
\hline Unnamed & 20 & 120 & $17,738, T \rightarrow C$ & $108, V \rightarrow A$ & & Chen et al (1991a) & 133 \\
\hline GER 9 & $<1$ & & $17,741, \mathrm{G} \rightarrow \mathrm{T}$ & $109, C \rightarrow F$ & & Knobloch \& Zoll & 649 \\
\hline HB111 & 7 & $<1$ & $17,743, T \rightarrow C$ & $110, S \rightarrow P$ & & Bottema et al (1991a) & 466 \\
\hline Taipei 5 & $<1$ & $<6$ & $17,746, T \rightarrow C$ & $111, C \rightarrow R$ & & Lin et al (1993) & 650 \\
\hline Calgary 24 & $<1$ & & $17,754,5$ or $6 \Delta 1$ & 114 or 115 & Frameshift & Poon et al (1993) & 651 \\
\hline Leamington & 13 & & $17,756, G \rightarrow C$ & $114, G \rightarrow A$ & & Ritchie et al (1989) & 134 \\
\hline Oxford e1 & 5 & 4 & $17,756, G \rightarrow C$ & $114, G \rightarrow A$ & & Winship \& Dragon (1991) & 135 \\
\hline Nastetten & $<1$ & & $17,759, A \rightarrow G$ & $115, Y \rightarrow C$ & & Ludwig et al & 136 \\
\hline UK 123 & 2 & 1 & $17,759, A \rightarrow G$ & $115, Y \rightarrow C$ & & Saad, Rowley et al & 467 \\
\hline Malmö 7 & $<1$ & $<0.1$ & $17,761, C \rightarrow T^{4}$ & $116, \mathrm{R} \rightarrow$ Stop & $\begin{array}{l}\text { Double (see 30,890), de } \\
\text { novo in MGF }\end{array}$ & Montandon et al (1990a) & 137 \\
\hline HB149 & $<1$ & 3 & $17,761, C \rightarrow T^{4}$ & $116, \mathrm{R} \rightarrow$ Stop & & Bottema et al (1991b) & 468 \\
\hline Taipei 6 & $<1$ & $<6$ & $17,761, C \rightarrow T^{4}$ & 116, R $\rightarrow$ Stop & & Lin et al (1993) & 652 \\
\hline UK 112 & $<1$ & & $17,761, C \rightarrow T^{4}$ & $116, \mathrm{R} \rightarrow$ Stop & & Saad, Rowley et al & 653 \\
\hline UK 28 & 5 & 5 & $17,761, C \rightarrow A$ & 116 , None & cryptic splice? & Green, Montandon et al & 138 \\
\hline Würzburg & $<1$ & & 17,764, Ins C & 117 & $\begin{array}{l}\text { Frameshift, de novo in } \\
\text { MGF(27) or MGM(21) }\end{array}$ & Ludwig et al (1992a) & 139 \\
\hline UK 9 & $<1$ & 0.4 & $17,773, \mathrm{~A} \rightarrow \mathrm{T}$ & $120, N \rightarrow Y$ & & Green et al (1989) & 140 \\
\hline GER 2228A & & & $17,778, G \rightarrow T$ & $121, Q \rightarrow H$ & & Wulff, Herrmann et al & 654 \\
\hline NZ 9 & 18 & & $17,778, G \rightarrow T$ & $121, Q \rightarrow H$ & & Van de Water et al & 655 \\
\hline UK 246 & $<1$ & & $17,782, T \rightarrow C$ & $123, S \rightarrow P$ & & Saad, Rowley et al & 656 \\
\hline HB88 & 2 & & $17,786, G \rightarrow A$ & $124, C \rightarrow Y$ & & Bottema et al (1991a) & 469 \\
\hline Taiwan & $<1$ & $<6$ & $17,795, C \rightarrow T$ & $127, A \rightarrow V$ & & Lin et al (1993) & 657 \\
\hline GER 10 & & & $17,796, A \rightarrow T$ & 127, None & Donor splice (e) & Knobloch \& Zoll & 658 \\
\hline GER 11 & & & 17,796, $A \rightarrow C$ & 127 , None & Donor splice (e) & Knobloch \& Zoll & 659 \\
\hline GER 37 & & & $17,796, A \rightarrow G$ & 127, None & Donor splice (e) & Knobloch \& Zoll & 660 \\
\hline HB68 & 1 & & $17,797, \mathbf{G} \rightarrow \mathrm{A}$ & $128, V \rightarrow M$ & Donor splice (e) & Bottema et al (1991a) & 470 \\
\hline Nörtingen & $<1$ & & $17,798, G \rightarrow T$ & & $\begin{array}{l}\text { Donor splice (e), } \\
\text { de novo in MGM(27) }\end{array}$ & Ludwig et al (1992a) & 141 \\
\hline Unnamed & $<1$ & $<1$ & $17,798, \mathrm{G} \rightarrow \mathrm{T}$ & & $\begin{array}{l}\text { Donor splice (e), de novo } \\
\text { in mother }\end{array}$ & Thompson et al (1992b) & 661 \\
\hline UK 102 & $<1$ & & $17,799, T \rightarrow G$ & Donor splice (e) & & Saad, Rowley et al & 662 \\
\hline Paris 5 & $<1$ & & $17,799, \mathrm{~T} \rightarrow \mathrm{C}$ & & $\begin{array}{l}\text { Donor splice (e), double } \\
\text { (see } 10,512 \text { ) }\end{array}$ & Ghanem et al (1993) & 639 \\
\hline (footnote 11) & $10^{11}$ & $5^{11}$ & $17,810, A \rightarrow G$ & & 6 cases. New donor splice? & $\begin{array}{l}\text { ?Ref Nos: }(53,69,102,116) \\
\text { PINs: }(142-4,664-6)\end{array}$ & \\
\hline GER 12 & & & $20,372, \mathrm{C} \rightarrow \mathrm{T}$ & $131, P \rightarrow L$ & & Knobloch \& Zoll & 667 \\
\hline Dakar & $<1$ & $<1$ & $20,374, T \rightarrow C$ & $132, C \rightarrow R$ & & Vidaud (1990) & 145 \\
\hline Taipei 7 & $<1$ & $<6$ & $20,374, T \rightarrow C$ & $132, \mathrm{C}-\mathrm{R}$ & & Lin et al (1993) & 668 \\
\hline JieLong 2 & $<1$ & $<6$ & $20,374, T \rightarrow C$ & $132, C \rightarrow R$ & & Lin et al (1993) & 669 \\
\hline Malmö 12 & $<1$ & $<0.1$ & $20,375, G \rightarrow T$ & $132, \mathrm{C} \rightarrow \mathrm{F}$ & & Green et al (1991b) & 146 \\
\hline GER 13 & $<1$ & & $20,375, G \rightarrow T$ & $132, \mathrm{C} \rightarrow \mathrm{F}$ & & Knobloch \& Zoll & 670 \\
\hline HB115 & $<1$ & & $20,375, G \rightarrow A$ & $132, C \rightarrow Y$ & Bottema et al (1991a) & 471 & \\
\hline ChangHua 1 & $<1$ & $<6$ & $20,375, G \rightarrow A$ & $132, \mathrm{C} \rightarrow \mathrm{Y}$ & & Lin et al (1993) & 671 \\
\hline UK 78 & $<1$ & & $20,375, G \rightarrow C$ & $132, \mathrm{C} \rightarrow \mathrm{S}$ & & Saad, Rowley et al & 672 \\
\hline Chapel Hill 2 & 4 & 4 & $20,377, \mathbf{G} \rightarrow \mathbf{A}$ & $133, \mathbf{G} \rightarrow \mathbf{R}$ & & High et al (1993) & 673 \\
\hline UK 166 & $<1$ & & $20,380-1, \Delta 2$ & 134 & Frameshift & Saad, Rowley et al & 674 \\
\hline HB145 & $<1$ & $<1$ & $20387-8 \Delta 2$ & 136 & $\begin{array}{l}\text { Frameshift, de novo in } \\
\text { MGF (32) }\end{array}$ & Ketterling et al. (1993) & 675 \\
\hline HB179 & 2 & $<1$ & $20387-8 \Delta 2$ & 136 & $\begin{array}{l}\text { Frameshift, haplotype } \\
\text { differs from HB145 de } \\
\text { novo in mother (30) }\end{array}$ & Ketterling et al. (1993) & 676 \\
\hline Malmö 44 & & & $20,398, \Delta 1$ & 140 & $\begin{array}{l}\text { Frameshift, de novo in } \\
\text { MGF or MGM }\end{array}$ & Green et al (1991b) & 147 \\
\hline (footnote 11) & $3^{11}$ & $41^{11}$ & $20,413, C \rightarrow T^{4}$ & $145, R \rightarrow C$ & $\begin{array}{l}16 \text { cases. Decreased XIa } \\
\text { activation }\end{array}$ & $\begin{array}{l}\text { Ref } N^{\text {os }}:(51,67,73,102, \\
116,135,137,141,143,152) \\
\text { PINs: }(148-52,472-6, \\
677-82)\end{array}$ & \\
\hline
\end{tabular}


Nucleic Acids Research, 1993, Vol. 21, No. 133083

$\begin{array}{lllll}\text { (footnote 11) } & 6^{11} & 113^{11} & 20,414, \mathrm{G} \rightarrow \mathrm{A}^{4} & 145, \mathrm{R} \rightarrow \mathrm{H} \\ & & & & \\ & & & & \\ & & & & \\ \text { Toronto } 21 & 7 & 60 & 20,414, \mathrm{G} \rightarrow \mathrm{T} & 145, \mathrm{R} \rightarrow \mathrm{L} \\ \text { HB23 } & <1 & & 20,466-78, \Delta 13 & 162 \\ & & & 20,497, \mathrm{C} \rightarrow \mathrm{T} & 173, \mathrm{Q} \rightarrow \mathrm{Stop} \\ \text { HB17 } & <1 & & 20,501, \Delta 1 & 174 \\ \text { HB78 } & 2 & <1 & 20,510, \Delta 1 & 177 \\ & & & 20,512, \mathrm{~T} \rightarrow \mathrm{C} & 178, \mathrm{~F} \rightarrow \mathrm{L} \\ \text { Malmö } 13 & <1 & 0.5 & 20,518, \mathrm{C} \rightarrow \mathrm{T}^{4} & 180, \mathrm{R} \rightarrow \mathrm{W} \\ \begin{array}{l}\text { Brest } \\ \text { (footnote } 11)\end{array} & <1 & 85 & & \end{array}$

17 cases. Decreased XIa Ref $N^{\text {os: }}(11-13,28,35$, activation $\quad 51,56,68,77,79,97,116$, $126,143)$ PINs: (153-62, 477-9, 684-7)

Also 9,786, A $\rightarrow C$ in intron Picketts et al (1990) 480

Frameshift, de novo in Koeberl et al (1989) 163 MGM (23)

Frameshift, de novo in mother (26)

Frameshift Ketterling et al (1993) Koeberl et al (1989) $\quad 164$

Double (see 20,518), N? Ghanem et al (1993) 167

11 cases including $5 \mathrm{~B}_{\mathrm{m}} \quad$ Ref $\mathrm{N}^{\mathrm{os}}$ : $(6,31,37,43,56$, $66,84,125,136)$

PINs: $(168-73,481-4$,

Brest

Madrid

$\begin{array}{ll}<1 & 85 \\ <1 & 90\end{array}$

$20,518, C \rightarrow G$

$180, R \rightarrow G$

$20,518, C \rightarrow G \quad 180, R \rightarrow G$

(footnote 11)

$<1^{11} \quad 112^{11}$

$20,519, \mathrm{G} \rightarrow \mathrm{A}^{4}$

$180, \mathrm{R} \rightarrow \mathrm{Q}$

Double (see 20,512)

690)

$\begin{array}{ll}\mathrm{B}_{\mathrm{m}} & \text { Solera et al }(1991) \\ 8 \text { cases including } 3 \mathrm{~B}_{\mathrm{m}} . & \text { Ref } \mathrm{N}^{\mathrm{os}} \text { : }(6,11,62,86,90,\end{array}$

Ghanem et al (1993)

decreased XIa activation $102,139,145)$

PINs: $(175-80,485,691)$

Taipei 8

Creston 1

Milano

Cardiff 2

UK 131

$<1$

73

$20,519, \mathrm{G} \rightarrow \mathrm{T}$

$180, \mathrm{R} \rightarrow \mathrm{L}$

$20,519, \mathrm{G} \rightarrow \mathrm{C} \quad 180, \mathrm{R} \rightarrow \mathrm{P}$

130

$20,521, G \rightarrow T$

$\begin{array}{ll}<1 & 130 \\ 15 & 132\end{array}$

$20,524, G \rightarrow C$

$20,524, G \rightarrow C$

$181, \mathrm{~V} \rightarrow \mathrm{FB}_{\mathrm{m}}$

$182, \mathrm{~V} \rightarrow \mathrm{L}$

$20,524, G \rightarrow T$

$182, \mathrm{~V} \rightarrow \mathrm{L}$

$20,525, \mathrm{~T} \rightarrow \mathrm{C}$

20,527-9, $\Delta 3$

$20,530, G \rightarrow A$

UK 54

Unnamed

Bottrop 1

UK 179

HB5 Japan

$20,531-3, \Delta 3$

$20,548, \mathrm{G} \rightarrow \mathrm{T}$

$182, \mathrm{~V} \rightarrow \mathrm{F}$

$182, \mathrm{~V} \rightarrow \mathrm{A} \quad \mathrm{B}_{\mathrm{m}}$

$\begin{array}{ll}<1 & 97 \\ <1 & 100\end{array}$

92

$183, \Delta \mathrm{G}$

$184, G \rightarrow R$

$184, \Delta \mathrm{G}$

$<1<1$

Unnamed

Seattle J

NanTou 1

Seattle $K$

Seattle L

Malmö 5

UK 114

UK 44

UK 233

Unnamed

Unnamed

Oxford 1

Rotenburg

UK 171

HB102

Calgary 22

UK 192

HB188

Paris 4

GER 16

UK 169

Toronto 19

UK 117

HB142

UK 43

Luanda

Unnamed

Taipei 9

UK 37

Wültschkau

Unnamed

Bottrop 2

HB72

Newcastle 1

Unnamed

TaiDung

Unnamed

HB41

Malmö 39

$<1$

$<1$

$190, \mathrm{G} \rightarrow \mathrm{C}$

$20,551, \mathrm{C} \rightarrow \mathrm{T}$

191, Q $\rightarrow$ Stop

20,551, C $\rightarrow$ A

191, Q $\rightarrow$ K

$20,553, A \rightarrow G \quad 191$, None

$20,554, T \rightarrow G$

$192, \mathrm{~F} \rightarrow \mathrm{V}$

$<6$

$20,560, T \rightarrow C$

$20,560, \mathrm{~T} \rightarrow \mathrm{A}$

$194, \mathrm{~W} \rightarrow \mathrm{R}$

$<1 \quad 1$

$20,561, G \rightarrow A$

20,561, G $\rightarrow$ A

$20,561, G \rightarrow A$

194, W $\rightarrow$ R

$194, \mathrm{~W} \rightarrow$ Stop

194, W $\rightarrow$ Stop

$194, \mathrm{~W} \rightarrow$ Stop

20,562, G $\rightarrow$ A $194, W \rightarrow$ Stop

20,563, C $\rightarrow \mathrm{T} \quad 195, \mathrm{Q} \rightarrow$ Stop

$<1$

$195, \mathrm{Q} \rightarrow$ Stop
$195, \mathrm{Q} \rightarrow \mathrm{R}$

$20,564, A \rightarrow G$
$20,566, G \rightarrow T$

$20,566, \mathrm{G} \rightarrow \mathrm{A}$

$30,038, \mathrm{G} \rightarrow \mathrm{A}$

$30,038, G \rightarrow C$

$<1$

19

$30,038, \mathrm{G} \rightarrow \mathrm{C}$

$30,046, \mathrm{~T} \rightarrow \mathrm{G}$

$30,046, \mathrm{~T} \rightarrow \mathrm{C}$

$30,058, \Delta 4$

$198, \mathrm{~L} \rightarrow \mathrm{W}$

$<1$

$<1$

$30,069, \mathrm{~T} \rightarrow \mathrm{C}$

$198, \mathrm{~L} \rightarrow \mathrm{S}$

202

$30,069, \mathrm{~T} \rightarrow \mathrm{C} \quad 206, \mathrm{C} \rightarrow \mathrm{R}$

$30,070, \mathrm{G} \rightarrow \mathrm{C} \quad 206, \mathrm{C} \rightarrow \mathrm{S}$

$30,070, G \rightarrow C \quad 206, C \rightarrow S$

$30,070, \mathrm{G} \rightarrow \mathrm{A} \quad 206, \mathrm{C} \rightarrow \mathrm{Y}$

$30,072, G \rightarrow A \quad 207, G \rightarrow R$

$30,072, G \rightarrow A \quad 207, G \rightarrow R$

$30,072, G \rightarrow T \quad 207, G \rightarrow$ Stop

$<1$

$\begin{array}{ll}<1 & <1 \\ <1 & 56\end{array}$

30,073

$30,076, \mathrm{G} \rightarrow \mathrm{A} \quad 208, \mathrm{G} \rightarrow \mathrm{D}$

$30,084, \mathrm{G} \rightarrow \mathrm{T} \quad 211, \mathrm{~V} \rightarrow \mathrm{F}$

$\begin{array}{ll}30,084, \mathrm{G} \rightarrow \mathrm{T} & 211, \mathrm{~V} \rightarrow \mathrm{F} \\ 30,090, \mathrm{G} \rightarrow \mathrm{T} & 213, \mathrm{E} \rightarrow \text { Stop }\end{array}$

$\begin{array}{llll}<1 & <1 & 30,09,, \mathrm{~T} \rightarrow \mathrm{T} & 213, \mathrm{E} \rightarrow \mathrm{S} \\ 4 & <1 & 30,096, \mathrm{~T} \rightarrow \mathrm{C} & 215, \mathrm{~W} \rightarrow \mathrm{R}\end{array}$

$30,096, T \rightarrow C \quad 215, W \rightarrow R$

$\begin{array}{llll}<1 & 0.1 & 30,097, \mathrm{G} \rightarrow \mathrm{A} & 215, \mathrm{~W} \rightarrow \text { Stop } \\ <1 & <6 & 30,098, \mathrm{G} \rightarrow \mathrm{T} & 215, \mathrm{~W} \rightarrow \mathrm{C}\end{array}$

$30,100, T \rightarrow C \quad 216, I \rightarrow T$

$30,100, T \rightarrow C \quad 216, I \rightarrow T$

$30,100, \mathrm{~T} \rightarrow \mathrm{C} \quad 216, \mathrm{I} \rightarrow \mathrm{T}$

$30,100, T \rightarrow C \quad 216, I \rightarrow T$

$\mathrm{B}_{\mathrm{m}}{ }^{6}$

Lin et al (1993) 692

Ritchie et al 486

181

Taylor et al (1990) 182

Saad, Rowley et al 693

Sakai et al (1989) 183

Nishimura et al (1990) 694

Maekawa et al (1991) 487

Green, Montandon et al 184

Thompson et al (1992b) 695

Ludwig et al $\quad 185$

Saad, Rowley et al $\quad 696$

Matsushita et al (1990)

Inhibitor, Double

(see -793)

Cryptic splice

Inhibitor

Normal carrier female

Chen et al (1991a)

Lin et al (1993) 697

Thompson et al (1992a) 489

Thompson et al (1992a) 490

Green et al (1989) $\quad 187$

Green, Montandon et al 188

Saad, Rowley et al 491

Saad, Rowley et al $\quad 698$

Thompson et al (1992b) 699

Ludwig et al 189

Donor splice (f) $\quad$ Rees et al (1985) $\quad 190$

Donor splice ( $\mathrm{f}$ Ludwig et al 191

Acceptor splice (g) Saad, Rowley et al 492

Acceptor splice (g) Bottema et al (1991a) 493

Acceptor splice (g), female Poon et al (1993) 700

Saad, Rowley et al 494

novo in MGF (21) Ketterling et al. (1993) 701

Frameshift

de novo in MGF

Ghanem et al (1993) 702

Knobloch \& Zoll $\quad 703$

Saad, Rowley et al $\quad 704$

Taylor et al (1990) 192

Saad, Rowley et al 705

Bottema et al (1991b) 495

Green, Montandon et al 193

David et al 706

Thompson et al (1992b) 707

Lin et al (1993) 708

Green, Montandon et al 194

Ludwig et al 195

Chen et al (1991a) $\quad 196$

Ludwig et al 197

Bottema et al (1990c) 198

496

Chen et al (1991a)

Lin et al (1993) 709

Chen et al (1991a) 200

Koeberl et al (1990a) 201

Green et al (1991b) 202

Knobloch \& Zoll $\quad 710$ 
3084 Nucleic Acids Research, 1993, Vol. 21, No. 13

HB65
Toronto 5 (HB36)
Toronto 11 (HB49)
Calgary 8
UK 93
HB24
Toronto 17 (HB58)
HB2
Unnamed
(footnote 11)

$\begin{array}{lllll}\text { Caen 3 } & <1 & <1 & 30,152, \text { Ins A } & 233 \\ \begin{array}{l}\text { Iceland 1 } \\ \text { Beauvais 1 }\end{array} & 3 & 119 & 30,800, \text { Ins A } & \text { None } \\ \text { HB6, Japan } & <1 & <1 & 30,800, \text { Ins A } & \text { None } \\ & & & 30,821, \mathrm{G} \rightarrow \mathrm{A} & \\ \text { Unnamed } & <1 & <1 & 30,821, \mathrm{G} \rightarrow \mathrm{A} & \\ \text { HB114 } & <1 & & 30,821, \mathrm{G} \rightarrow \mathrm{A} & \\ \text { UK 95 } & 6,10 & & 30,821, \mathrm{G} \rightarrow \mathrm{A} & \text { Female carrier } \\ \text { Spijkenisse } & 2 & 74 & 30,854, \mathrm{G} \rightarrow \mathrm{A} & 245, \mathrm{E} \rightarrow \mathrm{K} \\ \text { Monschau } & 3 & 39 & 30,855, \mathrm{~A} \rightarrow \mathrm{T} & 245, \mathrm{E} \rightarrow \mathrm{V} \\ \text { UK 140 } & <1 & & 30,857, \Delta 1 & 246 \\ \text { (footnote 11) } & <1^{11} & <1^{11} & 30,863, \mathrm{C} \rightarrow \mathrm{T}^{4} & 248, \mathrm{R} \rightarrow \text { Stop }\end{array}$

(footnote 11)

UK 92

(footnote 11)

15
$<1$
4
11
2
1
37
30
20
$14^{11}$

\section{3}

15

70

$13^{11}$
$30,101, T \rightarrow G$

$30,112, \mathrm{C} \rightarrow \mathrm{T}$

$30,112, \mathrm{C} \rightarrow \mathrm{T}$

$30,112, \mathrm{C} \rightarrow \mathrm{T}$

$30,117, \mathrm{~T} \rightarrow \mathrm{A}$

$30,119, \mathrm{~T} \rightarrow \mathrm{G}$

$30,134, \mathrm{~T} \rightarrow \mathrm{C}$

$30,134, \mathrm{~T} \rightarrow \mathrm{C}$

$30,134, T \rightarrow C$

$30,150, \mathrm{G} \rightarrow \mathrm{A}^{4}$

$216, \mathrm{I} \rightarrow \mathrm{M}$

$220, \mathrm{~A} \rightarrow \mathrm{V}$

$220, \mathrm{~A} \rightarrow \mathrm{V}$

$220, \mathrm{~A} \rightarrow \mathrm{V}$

$222, \mathrm{C} \rightarrow \mathrm{S}$

None

None

None

233, $\mathrm{A} \rightarrow \mathrm{T}$
$222, \mathrm{C} \rightarrow \mathrm{W}$

$30,864, G \rightarrow A^{4} \quad 248, R \rightarrow Q$

$30,873, \mathrm{~T} \rightarrow \mathrm{A} \quad 251, \mathrm{I} \rightarrow \mathrm{N}$

$<1$

$<1^{11}$

$<1^{11}$

$30,875, \mathrm{C} \rightarrow \mathrm{T}^{4}$

252, $\mathrm{R} \rightarrow$ Stop

UK 16

Malmö 7

HB131

13

$<1$

HB8

NZ 12

UK 88

GER 18

Malmö 50

UK 152

UK 138

Besanc̃on 2

HB208

UK 15

Beuren

HB96

Toronto 1 (HB31)

UK 75

UK 237

HB143

UK 48

San Antonio

HB101

Malmö 1

Zoeterwoude

Mantes-la-Jolie

Unnamed

GER 2234A

HB109

UK 58

Oxford h2

UK 13

UK 33

UK 41

UK 71

UK 239

HB100

24

1.5

$<1$

$<1$

$<1$

$<1$

$<1$

$<1$

16

16

20
$<1$

$<1$

5

$<1$

$<$

4

2

$<1$

$<1$

$<1$

13

$<1$

$<1$

$<1$

2

2

7

7

10

$<1$

14

$<1$

$<0$.

13

$<1$

$<1$

3

19

11

10

$<1$
$<0.1$

$<1$

$30,876, \mathrm{G} \rightarrow \mathrm{T} \quad 252, \mathrm{R} \rightarrow \mathrm{L}$ $30,890, \mathrm{C} \rightarrow \mathrm{T} \quad 257, \mathrm{H} \rightarrow \mathrm{Y}$ $30,897, A \rightarrow G \quad 259, Y \rightarrow C$

$30,900, A \rightarrow G \quad 260, N \rightarrow S$

$30,900, A \rightarrow G \quad 260, N \rightarrow S$

$30,924, A \rightarrow G \quad 268, H \rightarrow R$

$30,926, \mathrm{G} \rightarrow \mathrm{A} \quad 269, \mathrm{D} \rightarrow \mathrm{N}$

$30,927, A \rightarrow T \quad 269, D \rightarrow V$

$30,927, A \rightarrow G \quad 269, D \rightarrow G$

$30,927, A \rightarrow G \quad 269, D \rightarrow G$

$30,927, A \rightarrow G \quad 269, D \rightarrow G$

$30,928, \mathrm{C} \rightarrow \mathrm{A} \quad 269, \mathrm{D} \rightarrow \mathrm{E}$

$30,929, \mathrm{~A} \rightarrow \mathrm{T} \quad 270, \mathrm{I} \rightarrow \mathrm{F}$

$30,930, \mathrm{~T} \rightarrow \mathrm{C} \quad 270, \mathrm{I} \rightarrow \mathrm{T}$

$30,930, \mathrm{~T} \rightarrow \mathrm{C} \quad 270, \mathrm{I} \rightarrow \mathrm{T}$

$30,933, \mathrm{C} \rightarrow \mathrm{T} \quad 271, \mathrm{~A} \rightarrow \mathrm{V}$

$30,933, \mathrm{C} \rightarrow \mathrm{T} \quad 271, \mathrm{~A} \rightarrow \mathrm{V}$

$30,933, C \rightarrow T \quad 271, A \rightarrow V$

$30,936, T \rightarrow G \quad 272, \mathrm{~L} \rightarrow \mathrm{R}$

De novo in MGF (23)

$30,942, \Delta 1$

274

$30,945, \mathrm{~T} \rightarrow \mathrm{C}$

$275, \mathrm{~L} \rightarrow \mathrm{P}$
$30,945, \mathrm{~T} \rightarrow \mathrm{C} \quad 275, \mathrm{~L} \rightarrow \mathrm{P}$

$30,950-7, \Delta 8 \quad 277$

30,956,

277 T $\rightarrow$ A279, L $\rightarrow$ I

$30,981, \mathrm{C} \rightarrow \mathrm{T} \quad 287, \mathrm{P} \rightarrow \mathrm{L}$

$30,981, \mathrm{C} \rightarrow \mathrm{A} \quad 287, \mathrm{P} \rightarrow \mathrm{H}$

$30,985, T \rightarrow G \quad 288, I \rightarrow M$

$30,987, G \rightarrow T \quad 289, C \rightarrow F$

$30,992, \mathrm{G} \rightarrow \mathrm{C} \quad 291, \mathrm{~A} \rightarrow \mathrm{P}$

$30,992, \mathrm{G} \rightarrow \mathrm{A} \quad 291, \mathrm{~A} \rightarrow \mathrm{T}$

$30,992, \mathrm{G} \rightarrow \mathrm{A} \quad 291, \mathrm{~A} \rightarrow \mathrm{T}$

$30,992, \mathrm{G} \rightarrow \mathrm{A} \quad 291, \mathrm{~A} \rightarrow \mathrm{T}$

$30,992, \mathrm{G} \rightarrow \mathrm{A} \quad 291, \mathrm{~A} \rightarrow \mathrm{T}$

$30,992, \mathrm{G} \rightarrow \mathrm{A} \quad 291, \mathrm{~A} \rightarrow \mathrm{T}$

$31,001, \mathrm{G} \rightarrow \mathrm{T}$
$30,957, \mathrm{~T} \rightarrow \mathrm{C} \quad 279, \mathrm{~L} \rightarrow \mathrm{S}$

Triple (see 6575 \&

10,512),

de novo in MGF (29)

de novo mother (34)

carrier female

Frameshift, Inhibitor rameshift

15 cases including 1

inhibitor

12 cases

Same haplotype as 203

Double, (see 6,461), N

Double (see 6,461), N

Double (see 6,461), N

12 cases including 1 female Ref $\mathrm{N}^{\text {os: }}$ : $(20,51,68,79,86$,

carrier

Bottema et al (1991a)

497

Koeberl et al (1990a) 203

Koeberl et al (1990a) 204

Poon et al (1993) 711

Saad, Rowley et al $\quad 498$

Koeberl et al (1989) 205

Koeberl et al (1990a) 70

Koeberl et al (1989) 71

Chen et al (1991a) 72

116,129)

PINs: (206-14, 499 ,

712-3)

Ghanem et al (1993) 714

Double (see 31,119), N Green et al (1992a) 215

(1993)

715

Matsushita et al (1990) 216

Acceptor splice (h)

Acceptor splice (h)

Acceptor splice (h)

Chen et al (1991b) 217

Bottema et al (1991a) 500

Saad, Rowley et al $\quad 716$

Reitsma et al 218

Ludwig et al (1992b) 219

Saad, Rowley et al 501

Ref $\mathrm{N}^{\text {os }}:(13,15,48,51,52$,

$69,86,102,130,146$ )

PINs: $(220-9,502-5$

717)

20 cases including 1

Ref Nos: $(12,15,19,20,28$,

$43,69,85,102,116,139)$

PINs:(120, 230-3, 392,

$506-12,718-24)$

Saad, Rowley et al

Ref $N^{\text {os: }}(12,16,18,38,43$,

$51,52,66,101,116,119,131)$

PINs: (234-40, 513-4,

726-8)

Green, Montandon et al 241

Double (see 17,761), N

de novo in mother (38)

Proposed active site Asp

Proposed active site Asp

Proposed active site Asp

Proposed active site Asp

Montandon et al (1990a) 137

Bottema et al (1991b) 438

Ketterling et al (1993)

Koeberl et al (1989) 242

Van de Water et al 623

Green, Montandon et al 243

Knobloch \& Zoll $\quad 688$

Ljung et al $\quad 515$

Saad, Rowley et al 689

Saad, Rowley et al $\quad 729$

Ghanem et al (1993) 730

Ketterling et al. (1993) 731

Green, Montandon et al 244

Ludwig et al 245

Bottema et al (1991a) 516

Koeberl et al (1990a) 246

Saad, Rowley et al 732

Saad, Rowley et al 733

Bottema et al (1991b) 517

Ketterling et al (1993)

Green, Montandon et al 247

Frameshift, normal

Jagadeeswaran

Green et al (1989) 249

Reitsma et al 250

Ghanem et al (1993) 734

Chen et al (1991a) 251

Wulff, Herrmann et al 735

Bottema et al (1991a) 519

Saad, Rowley et al 736

Winship \& Dragon (1991) 252

Montandon et al (1989) 253

Green et al (1992a) 254

Green et al (1992a) 255

Saad, Rowley et al $\quad 520$

Saad, Rowley et al 737

De novo in MGM (24) Bottema et al (1991a) 521 


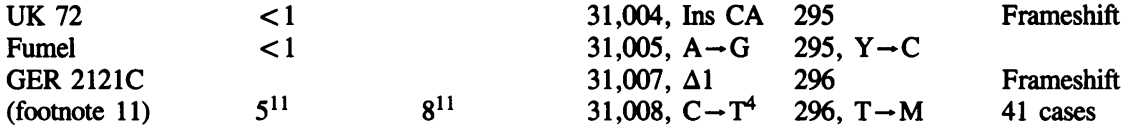

HB132

Malmö 18

Seattle 0

HB27

Unnamed

Seattle $\mathbf{P}$

Malmö 26

UK 122

Emsdetten

Oxford h3

Unnamed

Cenon

Toronto 21

GER 20

Unnamed

Albuquerque 2

NZ 6

GER 21

HB250

HB26

HB139

Amagasaki

UK 137

Kiryu

UK 11

Goldbach

UK 143

Toronto 7 (HB38)

$\begin{array}{ll}\text { UK } 147 & 4 \\ \text { NZ } 7 & 3\end{array}$

Bruxelles

Riegelsberg

HB82

ChangHua 2

HB122

Oxford h5

GER 22

Ursem

UK 90

Ratingen 2

GER 23

Oxford h1

Brünov

GER 24

NZ 10

(footnote 11)

$<1^{11}$

$<1$

$<1$

18

15

14

3

$<1$

$<1$

$<1$

$<1$

$<1$

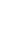

HB83

HB153

HB1 10

(footnote 11)

$\begin{array}{ll}31,012, \mathrm{C} \rightarrow \mathrm{T} & \text { None } \\ 31,035, \mathrm{G} \rightarrow \mathrm{A} & 305, \mathrm{G} \rightarrow \mathrm{D} \\ 31,039, \mathrm{~T} \rightarrow \mathrm{A} & 306, \mathrm{Y} \rightarrow \text { Stop } \\ 31,041, \mathrm{~T} \rightarrow \mathrm{C} & 307, \mathrm{~V} \rightarrow \mathrm{A} \\ 31,041, \mathrm{~T} \rightarrow \mathrm{C} & 307, \mathrm{~V} \rightarrow \mathrm{A} \\ 31,041, \mathrm{~T} \rightarrow \mathrm{C} & 307, \mathrm{~V} \rightarrow \mathrm{A} \\ 31,041, \mathrm{~T} \rightarrow \mathrm{G} & 307, \mathrm{~V} \rightarrow \mathrm{G} \\ 31,044, \mathrm{G} \rightarrow \mathrm{A} & 308, \mathrm{~S} \rightarrow \mathrm{N} \\ 31,045, \mathrm{~T} \rightarrow \mathrm{G} & 308, \mathrm{~S} \rightarrow \mathrm{R} \\ 31,046, \mathrm{G} \rightarrow \mathrm{A} & 309, \mathrm{G} \rightarrow \mathrm{S} \\ 31,047, \mathrm{G} \rightarrow \mathrm{T} & 309, \mathrm{G} \rightarrow \mathrm{V} \\ 31,047, \mathrm{G} \rightarrow \mathrm{A} & 309, \mathrm{G} \rightarrow \mathrm{D} \\ 31,049, \mathrm{~T} \rightarrow \mathrm{C} & 310, \mathrm{~W} \rightarrow \mathrm{R} \\ 31,050, \mathrm{G} \rightarrow \mathrm{T} & 310, \mathrm{~W} \rightarrow \mathrm{L} \\ 31,051, \mathrm{G} \rightarrow \mathrm{A} & 310, \mathrm{~W} \rightarrow \mathrm{Stop} \\ 31,051, \mathrm{G} \rightarrow \mathrm{A} & 310, \mathrm{~W} \rightarrow \mathrm{Stop} \\ 31,051, \mathrm{G} \rightarrow \mathrm{A} & 310, \mathrm{~W} \rightarrow \text { Stop } \\ 31,051, \mathrm{G} \rightarrow \mathrm{C} & 310, \mathrm{~W} \rightarrow \mathrm{C} \\ 31,051, \mathrm{G} \rightarrow \mathrm{C} & 310, \mathrm{~W} \rightarrow \mathrm{C} \\ 31,052, \mathrm{G} \rightarrow \mathrm{A} & 311, \mathrm{G} \rightarrow \mathrm{R} \\ 31,052, \mathrm{G} \rightarrow \mathrm{A} & 311, \mathrm{G} \rightarrow \mathrm{R} \\ 31,053, \mathrm{G} \rightarrow \mathrm{A} & 311, \mathrm{G} \rightarrow \mathrm{E} \\ 31,059, \mathrm{~T} \rightarrow \mathrm{G} & 313, \mathrm{~V} \rightarrow \mathrm{G} \\ 31,059, \mathrm{~T} \rightarrow \mathrm{A} & 313, \mathrm{~V} \rightarrow \mathrm{D} \\ 31,059-60, \Delta \mathbf{2} & 313 \\ 31,070, \mathrm{G} \rightarrow \mathrm{C} & 317, \mathrm{G} \rightarrow \mathrm{R} \\ 31,071, \mathrm{G} \rightarrow \mathrm{A} & 317, \mathrm{G} \rightarrow \mathrm{E} \\ 31,080, \mathrm{C} \rightarrow \mathrm{A} & 320, \mathrm{~A} \rightarrow \mathrm{D} \\ 31,080, \mathrm{C} \rightarrow \mathrm{A} & 320, \mathrm{~A} \rightarrow \mathrm{D} \\ 31,080, \mathrm{C} \rightarrow \mathrm{A} & 320, \mathrm{~A} \rightarrow \mathrm{D} \\ 31,080, \mathrm{C} \rightarrow \mathrm{T} & 320, \mathrm{~A} \rightarrow \mathrm{V} \\ 31,084-90, \Delta 7 & 321 \\ & \\ 31,091, \mathrm{C} \rightarrow \mathrm{T} & 324, \mathrm{Q} \rightarrow \text { Stop } \\ 31,092, \mathrm{~A} \rightarrow \mathrm{C} & 324, \mathrm{Q} \rightarrow \mathrm{P} \\ 31,096, \mathrm{C} \rightarrow \mathrm{G} & 325, \mathrm{Y} \rightarrow \mathrm{Stop} \\ 31,103, \mathrm{G} \rightarrow \mathrm{T} & 328, \mathrm{~V} \rightarrow \mathrm{F} \\ 31,103, \mathrm{G} \rightarrow \mathrm{T} & 328, \mathrm{~V} \rightarrow \mathrm{F} \\ 31,103, \mathrm{G} \rightarrow \mathrm{A} & 328, \mathrm{~V} \rightarrow \mathrm{I} \\ 31,110, \mathrm{~T} \rightarrow \mathrm{C} & 330, \mathrm{~L} \rightarrow \mathrm{P} \\ 31,110-12, \Delta 3^{9} & 331, \Delta \mathrm{V} \\ 31,110-12, \Delta 3^{9} & 331, \Delta \mathrm{V} \\ 31,113, \mathrm{~T} \rightarrow \mathrm{C} & 331, \mathrm{~V} \rightarrow \mathrm{A} \\ 31,115, \mathrm{G} \rightarrow \mathrm{T} & 332, \mathrm{D} \rightarrow \mathrm{Y} \\ 31,115, \mathrm{G} \rightarrow \mathrm{T} & 332, \mathrm{D} \rightarrow \mathrm{Y} \\ 31,115, \mathrm{G} \rightarrow \mathrm{T} & 332, \mathrm{D} \rightarrow \mathrm{Y} \\ 31,118, \mathrm{C} \rightarrow \mathrm{T}^{4} & 333, \mathrm{R} \rightarrow \text { Stop } \\ & \end{array}$

$31,118, C \rightarrow G$ $31,118, C \rightarrow G$ $31,119, \mathrm{G} \rightarrow \mathrm{T}$ $31,119, \mathrm{G} \rightarrow \mathrm{A}^{4}$

333, $R \rightarrow G$ $333, R \rightarrow G$
333, $\mathrm{R} \rightarrow \mathrm{L}$
$<1$ $2^{11}$ 63 $88^{11}$

70
63
150
$88^{11}$

Ketterling et al (1993) 738

Saad, Rowley et al 739

Ghanem et al (1993) 740

Wulff, Herrmann et al 741

Ref $\mathrm{N}^{\mathrm{os}}:(20,49,51,52,59$,

$64-5,67-9,79,86,102$,

$114,116,130,136,139$.

$143,152)$

PINs: $(256-74,393-4$,

$522-31,742-52$ )

Double (see 31,224) N Bottema et al (1991b) 532

de novo in MGF Green et al (1991b) 275

Thompson et al (1992a) 533

Bottema et al (1989a) 276

Chen et al (1991a) 277

Same haplotype as $277 \quad$ Thompson et al (1992a) 534

Green et al (1991b) 278

Green, Montandon et al 279

Ludwig et al 280

Winship \& Dragon (1991) 281

Thompson et al (1989) 282

Ghanem et al (1993) 753

Picketts et al 283

Knobloch \& Zoll $\quad 754$

Wang et al (1990a) 284

Tarnower \& Smith (1991) 285

Van de Water et al 535

de novo in mother (28) Knobloch \& Zoll 755

de novo MGF (22) Ketterling et al (1993) 756

Koeberl et al (1989) 286

Same haplotype as $286 \quad$ Bottema et al (1991b) 536

B $^{6} \quad$ Miyata et al (1991) 287

Green, Montandon et al 288

$\mathrm{B}_{\mathrm{m}}$

Green et al (1989) 289

Ludwig et al 290

Female carrier $\quad$ Saad, Rowley et al $\quad 758$

Koeberl et al (1990a) 291

Saad, Rowley et al 537

Van de Water et al $\quad 538$

Ghanem et al (1993) 758

Frameshift, double (see 37), Ludwig et al (1992a) 14

de novo in mother(30)

Bottema et al (1991a) 539

Lin et al (1993) 759

De novo in mother (26) Bottema et al (1991a) 540

Winship (1990) 292

$\begin{array}{lll}\text { de novo in MGF } & \text { Knobloch \& Zoll } & 760 \\ \text { Double (see 6,491-4), N } & \text { Poort et al (1990) } & 77\end{array}$

Green, Montandon et al 293

In frame Ludwig et al 294

In frame, de novo in Knobloch \& Zoll 761

mother (19)

Winship \& Dragon (1991) 295

Same haplotype as GER 24 Ludwig et al 296

Same haplotype as $296 \quad$ Knobloch \& Zoll 762

Van de Water et al 763

16 cases including 1 Ref $\mathrm{N}^{\mathrm{os}}$ : $(12,20,28,43,66$,

female carrier $\quad 70,77,79,86,93,106,116)$

PINs: (297-303, 541-3,

764-9)

Bottema et al (1990c) 304

Haplotype differs from 304 Bottema et al (1991b) 544

Bottema et al (1991a) 545

19 cases including 1 Ref $N^{\circ s}$ : $(12,20,28,43,48$,

female carrier, and $49,52,84,86,99$,

double (see 30,800) )

$102,103,116,136,142,146)$

PINs: $(215,305-15$,

$546-8,770-3)$

Chan et al (1991) $\quad 316$

Ljung et al 774

Green et al (1989) $\quad 317$

Saad, Rowley et al $\quad 549$

Chen et al (1991a) $\quad 318$

Saad, Rowley et al $\quad 775$

Green et al (1991b) 319

Knobloch \& Zoll 776 
3086 Nucleic Acids Research, 1993, Vol. 21, No. 13

\begin{tabular}{|c|c|c|c|c|c|c|c|}
\hline $\begin{array}{l}\text { Calgary } 3 \\
\text { (footnote 11) }\end{array}$ & ${ }^{4}<1^{11}$ & $\begin{array}{l}120 \\
<1^{11}\end{array}$ & $\begin{array}{l}31,130, C \rightarrow A \\
31,133, C \rightarrow T^{4}\end{array}$ & $\begin{array}{l}337, \mathrm{~L} \rightarrow \mathrm{I} \\
338, \mathrm{R} \rightarrow \text { Stop }\end{array}$ & $\begin{array}{l}10 \text { cases including } 1 \\
\text { double (see } 48 \text { ) }\end{array}$ & $\begin{array}{l}\text { Fraser et al }(1992) \\
\text { Ref } N^{\text {os: }}(36,41,49,78,82 \text {, } \\
101,129) \\
\text { PINs: }(320-6,399 \text {, } \\
777-8)\end{array}$ & 550 \\
\hline GER 26 & 10 & & $31,134, \mathrm{G} \rightarrow \mathrm{C}$ & $338, \mathrm{R} \rightarrow \mathrm{P}$ & & Knobloch \& Zoll & 779 \\
\hline HB241 & $<1$ & & $31,140, C \rightarrow G$ & $340, T \rightarrow R$ & de novo in MGF (27) & Ketterling et al. (1993) & 780 \\
\hline UK 170 & & & $\begin{array}{l}31,141,2 \text { or } 3 \\
\text { Ins AA }\end{array}$ & 340 or 341 & $\begin{array}{l}\text { Frameshift, normal } \\
\text { carrier female }\end{array}$ & Saad, Rowley et al & 551 \\
\hline Samli & $<1$ & $<1$ & $\begin{array}{l}31,149-51, \Delta 3 \\
31,158-62, \Delta 5 \\
31,158, \text { Ins G }\end{array}$ & 343 & Frameshift & Ludwig et al & 327 \\
\hline Gladbeck & 4 & 87 & $31,151, \mathrm{~A} \rightarrow \mathrm{T}$ & $344, I \rightarrow F$ & & Ludwig et al & 328 \\
\hline Unnamed & $<1$ & $<1$ & $\begin{array}{l}31,157,8 \text { or } 9 \\
\text { Ins AA }\end{array}$ & 346 & Frameshift & Chen et al (1991b) & 329 \\
\hline Seattle $\mathbf{R}$ & $<1$ & $<1$ & $\begin{array}{l}31,157,8 \text { or } 9 \\
\text { Ins AA }\end{array}$ & 346 & Frameshift & Thompson et al (1992a) & 552 \\
\hline UK 135 & & & $\begin{array}{l}31,157-9 \text { or } \\
31,160-2 \Delta 3\end{array}$ & $\begin{array}{l}346 \text { or } 347 \\
\Delta \mathrm{N}\end{array}$ & In frame & Saad, Rowley et al & 553 \\
\hline HB108 & $<1$ & 2 & $31,161, \mathrm{~A} \rightarrow \mathrm{T}$ & $347, \mathrm{~N} \rightarrow \mathrm{I}$ & & Bottema et al (1991a) & 554 \\
\hline Unnamed & 3 & 103 & $31,163, A \rightarrow G$ & $348, M \rightarrow V$ & & Chen et al (1991a) & 330 \\
\hline HB124 & $<1$ & 40 & $31,165, \mathrm{G} \rightarrow \mathrm{A}$ & $348, M-I$ & De novo in MGF (28) & $\begin{array}{l}\text { Bottema et al (1991a) } \\
\text { Ketterling et al (1993) }\end{array}$ & 555 \\
\hline Offenbach & $<1$ & & 31,166 or $7, \Delta 1$ & 349 & $\begin{array}{l}\text { Frameshift, de novo in } \\
\text { MGM(29) }\end{array}$ & Ludwig et al (1992a) & 331 \\
\hline Kingston 4 & 35 & 45 & $31,170, G \rightarrow C$ & $350, C \rightarrow S$ & Somatic mosaic & Taylor et al (1991) & 332 \\
\hline UK 17 & $<1$ & $<1$ & & $31,170, G \rightarrow A$ & $350, \mathrm{C} \rightarrow \mathrm{Y}$ & Green, Montandon et al & 333 \\
\hline Malmö 47 & $<1$ & & $31,170, G \rightarrow A$ & $350, \mathrm{C} \rightarrow \mathrm{Y}$ & & Ljung et al & 556 \\
\hline GER 27 & $<1$ & & $31,194-5,2$ & 358 & $\begin{array}{l}\text { Frameshift, de novo } \\
\text { in mother (18) }\end{array}$ & Knobloch \& Zoll & 781 \\
\hline GER 28 & 1.5 & & $31,197, A \rightarrow G$ & $359, \mathrm{D} \rightarrow \mathrm{G}$ & & Knobloch \& Zoll & 782 \\
\hline Unnamed & 2 & 130 & $31,200, \mathrm{C} \rightarrow \mathrm{T}$ & $360, S \rightarrow L$ & & Chen et al (1991a) & 334 \\
\hline UK 164 & 1 & & $31,200, \mathrm{C} \rightarrow \mathrm{T}$ & $360, S \rightarrow L$ & & Saad, Rowley et al & 783 \\
\hline Bordeaux 2 & $<1$ & & $31,200, \mathrm{C} \rightarrow \mathrm{T}$ & $360, S \rightarrow L$ & & Ghanem et al (1993) & 784 \\
\hline Poissy & 1.5 & & $31,200, C \rightarrow G$ & $360, S \rightarrow$ Stop & & Ghanem et al (1993) & 785 \\
\hline Modane & $<1$ & & $31,200, \mathrm{C} \rightarrow \mathrm{G}$ & $360, S \rightarrow$ Stop & & Ghanem et al (1993) & 786 \\
\hline Madrid 5 & $<1$ & $<1$ & $31,202, \mathrm{~T} \rightarrow \mathrm{C}$ & $361, \mathrm{C} \rightarrow \mathrm{R}$ & & Damas et al & 787 \\
\hline UK 144 & $<1$ & & $31,208, \mathrm{G} \rightarrow \mathrm{T}$ & $363, \mathrm{G} \rightarrow$ Stop & & Saad, Rowley et al & 557 \\
\hline Los Angeles 2 & $<1$ & 110 & $31,208, G \rightarrow A$ & $363, G \rightarrow R$ & & High et al (1993) & 558 \\
\hline Eagle Rock & $1-5$ & 100 & $31,209, \mathrm{G} \rightarrow \mathrm{T}$ & $363, \mathrm{G} \rightarrow \mathrm{V}$ & & Bajaj et al (1990) & 335 \\
\hline Seattle $S$ & 3 & 100 & $31,209, \mathrm{G} \rightarrow \mathrm{C}$ & $363, \mathrm{G} \rightarrow \mathrm{A}$ & De novo in MGF & Thompson et al (1992a) & 559 \\
\hline UK 35 & 2 & 53 & $31,209, \mathrm{G} \rightarrow \mathrm{A}$ & $363, \mathrm{G} \rightarrow \mathrm{E}$ & & Green, Montandon et al & 336 \\
\hline NZ 8 & $<1$ & & $31,209, \mathrm{G} \rightarrow \mathrm{A}$ & $363, \mathrm{G} \rightarrow \mathrm{E}$ & & Van de Water et al & 788 \\
\hline Mechtal & $<1$ & 100 & $31,211, \mathrm{G} \rightarrow \mathrm{C}$ & $364, \mathrm{D} \rightarrow \mathrm{H}$ & & Ludwig et al (1992b) & 337 \\
\hline HB80 & 2 & 95 & $31,211, G \rightarrow C$ & $364, D \rightarrow H$ & & Bottema et al (1990c) & 338 \\
\hline UK 30 & 2 & & $31,211, \mathrm{G} \rightarrow \mathrm{A}$ & $364, \mathrm{D} \rightarrow \mathrm{N}$ & $\mathrm{B}_{\mathrm{m}}$ & Green, Montandon et al & 339 \\
\hline Unnamed & $<1$ & 130 & $31,212, \mathrm{~A} \rightarrow \mathrm{T}$ & $364, D \rightarrow V$ & De novo in MGF & Chen et al (1991a) & 340 \\
\hline Varel & $<1$ & 89 & $\begin{array}{l}31,213-14 \\
\mathrm{TA} \rightarrow \mathrm{CG}\end{array}$ & $365, S \rightarrow G$ & $\begin{array}{l}\text { Active site, } \\
\text { Inhibitor, silent } \\
\text { mutation at aa364 }\end{array}$ & Ludwig et al $(1988,1992 b)$ & 341 \\
\hline GER 29 & & & $31,214, A \rightarrow G$ & $365, S \rightarrow G$ & Active site & Knobloch \& Zoll & 789 \\
\hline Schmallenberg & $<1$ & & $31,215, \mathrm{G} \rightarrow \mathrm{T}$ & $365, S \rightarrow I$ & Active site & Ludwig et al (1992b) & 342 \\
\hline UK 162 & 1.8 & & $31,215, \mathrm{G} \rightarrow \mathrm{T}$ & $365, S \rightarrow I$ & Active site & Saad, Rowley et al & 790 \\
\hline Toronto 4 (HB35) & 1 & 90 & $31,216, T \rightarrow A$ & $365, S \rightarrow R$ & Active site & Koeberl et al (1990a) & 343 \\
\hline KaoHsiung 2 & $<1$ & 100 & $31,216, T \rightarrow G$ & $365, S \rightarrow R$ & Active site & Lin et al & 791 \\
\hline HB87 & $<1$ & 32 & $31,218, \mathrm{G} \rightarrow \mathrm{A}$ & $366, \mathrm{G} \rightarrow \mathrm{E}$ & & Bottema et al (1991a) & 560 \\
\hline Limoges & 6 & & $31,218, \mathrm{G} \rightarrow \mathrm{A}$ & $366, \mathrm{G} \rightarrow \mathrm{E}$ & & Ghanem et al (1993) & 792 \\
\hline Unnamed & $<1$ & 14 & $31,220, \mathrm{G} \rightarrow \mathrm{A}$ & $367, \mathrm{G} \rightarrow \mathrm{R}$ & & Chen et al (1991a) & 344 \\
\hline HB300 & 3 & & $31,220, \mathrm{G} \rightarrow \mathrm{A}$ & $367, \mathrm{G} \rightarrow \mathrm{R}$ & & Caglayan et al & 793 \\
\hline Chambéry & $<1$ & & $31,220, \mathrm{G} \rightarrow \mathrm{A}$ & $367, G \rightarrow R$ & & Ghanem et al (1993) & 794 \\
\hline Bergamo & $<1$ & 156 & $31,223, \mathrm{C} \rightarrow \mathrm{A}$ & $368, \mathrm{P} \rightarrow \mathrm{T}$ & $\mathrm{B}_{\mathrm{m}}$ & Bertina et al (1990) & 345 \\
\hline Calgary 20 & 7 & $>100$ & $31,223, \mathrm{C} \rightarrow \mathrm{A}$ & $368, P \rightarrow T$ & & Poon et al (1993) & 795 \\
\hline UK 190 & 3 & & $31,224, \mathrm{C} \rightarrow \mathrm{T}$ & $368, P \rightarrow L$ & & Saad, Rowley et al & 561 \\
\hline HB132 & & & $31,224, \mathrm{C} \rightarrow \mathrm{T}$ & $368, P \rightarrow L$ & Double (see 31,012 ) & Bottema et al (1991b) & 532 \\
\hline HB73 & $<1$ & $<1$ & $31,227, A \rightarrow G$ & $369, \mathrm{H} \rightarrow \mathrm{R}$ & & Bottema et al $(1990 c)$ & 346 \\
\hline Malmö 16 & 15 & 13 & $31,248, \mathrm{C} \rightarrow \mathrm{A}$ & $376, T \rightarrow N$ & & Green et al (1991b) & 347 \\
\hline Unnamed & $<1$ & $<1$ & $31,253, T \rightarrow C$ & $378, \mathrm{~F} \rightarrow \mathrm{L}$ & & Chen et al (1991a) & 348 \\
\hline GER 30 & $<1$ & & $31,257, \mathrm{~T} \rightarrow \mathrm{G}$ & $379, \mathrm{~L} \rightarrow$ Stop & de novo in mother & Knobloch \& Zoll & 796 \\
\hline Brantford & 5 & & $31,258, A \rightarrow C$ & $379, \mathrm{~L} \rightarrow \mathrm{F}$ & & Tam et al (1991) & 349 \\
\hline Barcelos & $<1$ & & $31,259, \mathrm{~A} \rightarrow \mathrm{C}$ & $380, T \rightarrow P$ & & David et al & 562 \\
\hline Hong Kong 5 & 7 & $<10$ & $31,260, C \rightarrow G$ & $380, T \rightarrow S$ & & Chan et al (1991) & 350 \\
\hline UK 76 & $<1$ & 1 & $31,260, \mathrm{C} \rightarrow \mathrm{T}$ & $380, \mathrm{~T} \rightarrow \mathrm{I}$ & & Green, Montandon et al & 351 \\
\hline Hong Kong 4 & $<1$ & $<10$ & $31,261, \Delta 1$ & 380 & Frameshift & Chan et al (1991) & 352 \\
\hline GER 31 & $<1$ & & 31,270, Ins $T$ & 383 & Frameshift & Knobloch \& Zoll & 797 \\
\hline GER 32 & $<1$ & & $31,274, T \rightarrow C$ & $385, \mathrm{~W} \rightarrow \mathrm{R}$ & & Knobloch \& Zoll & 798 \\
\hline Malmö 49 & $<1$ & & $31,276, \mathrm{G} \rightarrow \mathrm{A}$ & $385, \mathrm{~W} \rightarrow$ Stop & & Ljung et al & 563 \\
\hline Takatsuki & 2 & 120 & $31,278, \mathrm{G} \rightarrow \mathrm{A}$ & $386, G \rightarrow D$ & $\mathrm{~B}_{\mathrm{m}}{ }^{6}$ & Kuze et al (1992) & 799 \\
\hline
\end{tabular}




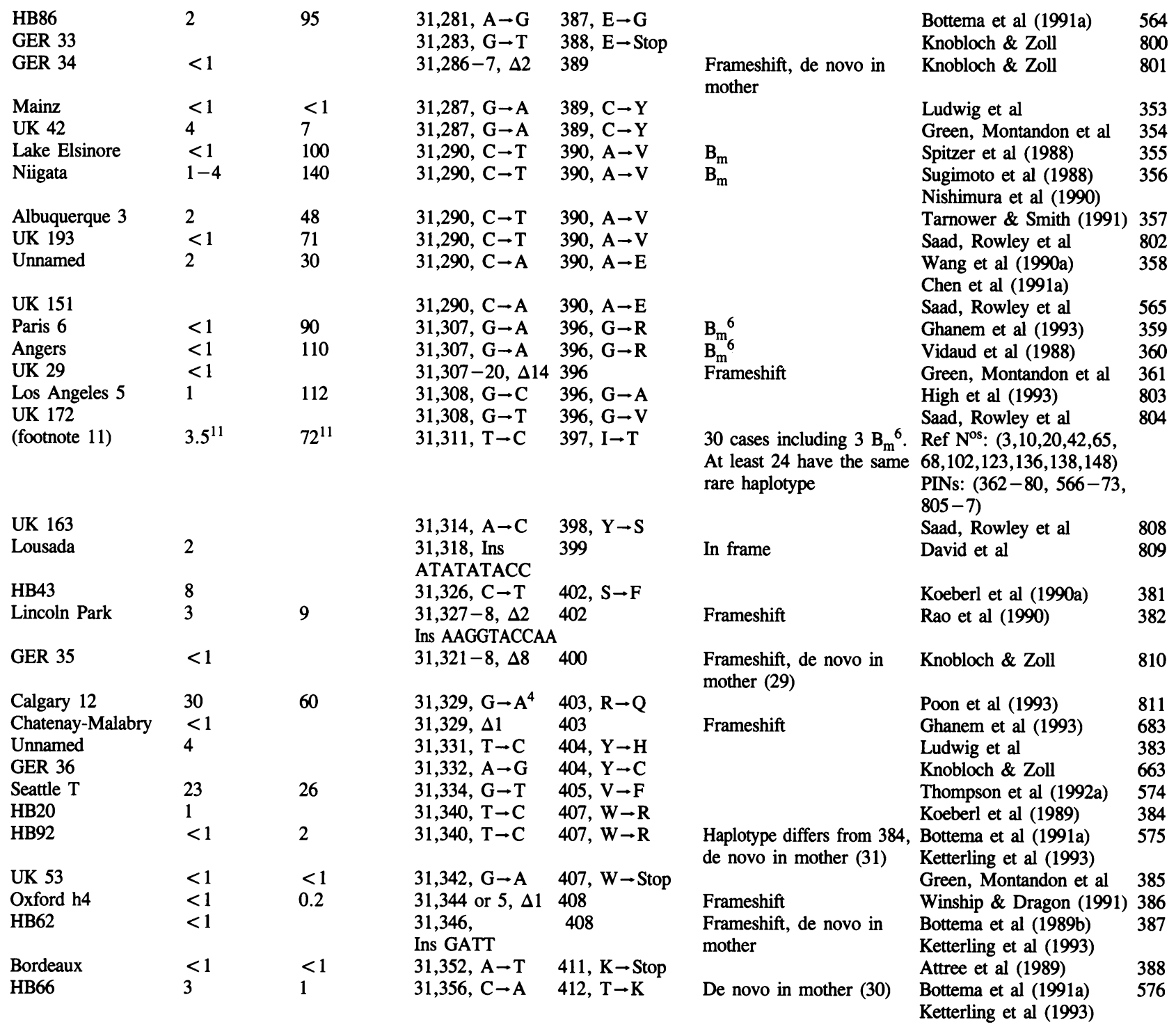

${ }^{1}$ For nucleotide numbering see Yoshitake et al (1985); for amino acid numbering Anson et al (1984).

2 Nucleotide change predicted from amino acid sequence.

3 The following comments or abbreviations are used:

(i) Inhibitor-patients developing anti-factor IX antibodies in response to therapeutic factor IX.

(ii) Frameshift-caused by the addition (symbol Ins) or deletion (symbol $\Delta$ ) affecting nucleotides corresponding to the stated amino acid number and terminating at a new stop codon shortly after.

(iii) Double-a double mutant, entered twice in the data base and cross-referenced.

(iv) $\mathrm{N}$-indicates the mutation, usually a double mutant, is probably a normal variant-not causing the disease.

(v) the exon $(\mathrm{a}-\mathrm{h})$ immediately adjacent to donor or acceptor splice sites is noted.

(vi) Gla refers to glutamic acid residues normally $\gamma$-carboxylated, and $\beta$-hydroxyaspartate to the single modified aspartate residue.

(vii) $B_{m}$-patients with a prolonged bovine prothrombin time (Hougie \& Twomey, 1967).

(viii) de novo-refers to a new mutation originating in mother, or maternal grandmother (MGM)-mother, but not grandmother carrying the mutation, and RFLP analysis indicating the mutant gene is inherited from the grandmother, or maternal grandfather (MGF)-mother but not grandparents carrying the mutation and RFLP analysis indicating the mutant gene is inherited from the grandfather. Parental age at conception of child carrying, or affected by, the mutant gene is given in brackets, if known.

4 Indicates mutation of a CG to either TG or CA.

$5 \%$ varies with age, rising after puberty.

6 Bovine prothrombin time is moderately prolonged.

7 Patients are uniquely named, except for 'Vancouver,Fr' and 'Angers', where the authors have not distinguished different patients with the same mutation.

8 The position of insertions (Ins) corresponds to the first nucleotide of the inserted base. E.g. In Recklinghausen,

114 , Ins AT, patient identity number $=17$, the inserted dinucleotide AT occupies nucleotides 114 and 115 and displaces the residues normally found there, which now become residues 116 and 117

9 Or, $31,111-3$, or $31,112-4$, or $31,113-5$.

10 The PIN number is a patient identification number, used as an aid to patient identification now and in the future. It is not the intention that the PIN number should replace any existing nomenclature; rather, it will co-exist with it. Once a patient (and patients within the same pedigree) is given a PIN number, this will remain unaltered in updated, or new versions of this database. The reason for the slightly higher number of PINS (576) than patient entries (574) is because of amalgation of separate entries. This arose as originally separate studies are now known to refer to one and the same patient, or to patients related by pedigree. 11 Mutations which have recurred more than five times have been summarized. Factor IX clotting and antigen levels have been entered as an average of the available figures. The comments column gives the number of repeats reported plus any further comments that may relate to individual patients. 